\begin{abstract}
A DYNAMIC TAXI RIDE SHARING SYSTEM USING PARTICLE SWARM OPTIMIZATION
\end{abstract}

by Shrawani Silwal

With the rapid growth of on-demand taxi services, like Uber, Lyft, etc., urban public transportation scenario is shifting towards a personalized transportation choice for most commuters. While taxi rides are comfortable and time efficient, they often lead to higher cost and road congestion due to lower overall occupancy than bigger vehicles. A possible solution to improve taxi occupancy is to adopt ride sharing. Existing ride sharing solutions are mostly centralized and proprietary. However, given the wide spatio-temporal variation of incoming ride requests designing a dynamic and distributed shared-ride scheduling system is NP-hard. In this thesis, we have proposed a publisher (passengers) and subscriber (taxis) based ride sharing system that provides effective real-time ride scheduling for multiple passengers willing to share rides in part or in full. A particle swarm based route optimization strategy has been applied to determine the most preferable route for passengers. Empirical analysis using large scale single-user taxi ride records from Chicago Transit Authority, show that, our proposed system, ensures a maximum of $91.74 \%$ and $63.29 \%$ overall success rates during peak and non-peak hours, respectively. 
Thesis

\author{
Submitted to the \\ Faculty of Miami University \\ in partial fulfillment of \\ the requirements for the degree of \\ Master of Science \\ by \\ Shrawani Silwal \\ Miami University \\ Oxford, Ohio \\ 2020 \\ Advisor: Dr.Vaskar Raychoudhury \\ Reader: Dr.Karen Davis \\ Reader: Dr.Md Osman Gani
}

C2020 Shrawani Silwal 
This thesis titled

\section{A DYNAMIC TAXI RIDE SHARING SYSTEM USING PARTICLE SWARM OPTIMIZATION}

by

Shrawani Silwal

has been approved for publication by

The College of Engineering and Computing

and

The Department of Computer Science \& Software Engineering

Dr.Vaskar Raychoudhury

Dr.Karen Davis

Dr.Md Osman Gani 


\section{Table of Contents}

$\begin{array}{ll}\text { List of Tables } & \mathbf{v}\end{array}$

List of Figures $\quad$ vi

Dedication viii

$\begin{array}{ll}\text { Acknowledgements } & \text { ix }\end{array}$

1 Chapter 1 :Introduction $\quad 1$

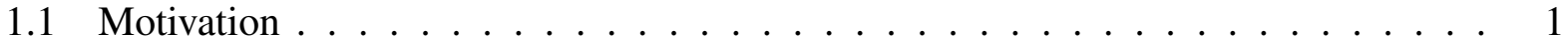

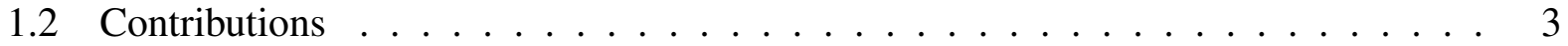

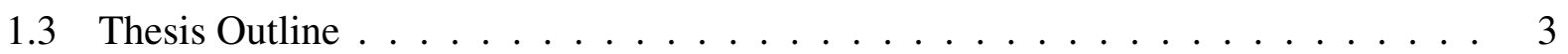

2 Chapter 2 : Background \& Related Work 4

2.1 Introduction to Optimization . . . . . . . . . . . . . . 4

2.2 Particle Swarm Optimization (PSO) . . . . . . . . . . . . . . 5

2.3 Types of Ride Sharing Systems . . . . . . . . . . . . . . . . . 5

2.3.1 Static Ride Sharing . . . . . . . . . . . . . . . . 5

2.3.2 Dynamic Ride Sharing . . . . . . . . . . . . . . . . 8

2.4 Dynamic Ride Sharing Architecture . . . . . . . . . . . . . . . . . 8

2.4.1 Centralized Ride Sharing . . . . . . . . . . . . . . . . . 9

2.4.2 Distributed Ride Sharing . . . . . . . . . . . . . . . . . . 10

2.4.3 Hybrid Ride Sharing . . . . . . . . . . . . . . . . . . . . 10

2.5 Related Work . . . . . . . . . . . . . . . . . . . . 12

2.6 Conclusion . . . . . . . . . . . . . . . . . . . 14

3 Chapter 3 : Data Set and Pre-Processing 16

3.1 Data Pre-processing . . . . . . . . . . . . . . . . 16

3.2 Data Analysis . . . . . . . . . . . . . . . . . . . . . 17

3.2.1 Temporal Analysis of the Pick-Up Requests . . . . . . . . . . . . . . . 21

3.2.2 Spatial Analysis of the Pick-Up Request in Chicago City . . . . . . . . . . 22

4 Chapter 4 : Proposed Method: Dynamic Ride Sharing System using Particle Swarm $\begin{array}{ll}\text { Optimization } & 24\end{array}$

4.1 Pseudo Code . . . . . . . . . . . . . . . . . . . . . . . 25 
4.1.1 Pseudo Code at Passenger's End . . . . . . . . . . . . . . . . . 25

4.1.2 Pseudo Code at Taxi's End . . . . . . . . . . . . . . . . . 27

4.1.3 PSO for Route Optimization . . . . . . . . . . . . . . . . 27

5 Chapter 5 : Results and Validation $\quad 30$

5.1 Experimental Design . . . . . . . . . . . . . . . . . 30

5.2 Performance Metrics . . . . . . . . . . . . . . . . . . . . . . . 32

5.3 Results for Summer Week and Results for Winter Week . . . . . . . . . . . . . . 34

5.3 .1 Results for Summer Week . . . . . . . . . . . . . . . . 34

5.3 .2 Results for Winter Week . . . . . . . . . . . . . . . . . . . . . . . . . . . . . . . . . . .

5.4 Summary of the observations . . . . . . . . . . . . . . . . . . 45

5.5 Comparison of Results . . . . . . . . . . . . . . . . . . . . 46

6 Chapter 6 : Conclusion and Future Works $\quad 48$

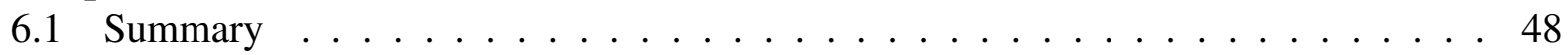

6.2 Future Works . . . . . . . . . . . . . . . . . 48

$\begin{array}{lr}\text { References } & 49\end{array}$ 


\section{List of Tables}

2.1 Pros and Cons of different RSS architecture [1] . . . . . . . . . . . . . 12

2.2 Ride-Share Variants [2] . . . . . . . . . . . . . . . . . . . 13

2.3 Related Work Comparison . . . . . . . . . . . . . . . 15

3.1 Few columns from the Dataset . . . . . . . . . . . . . . 16

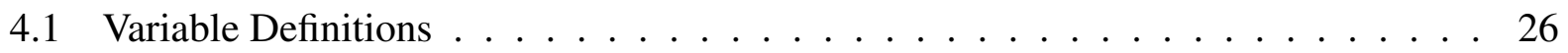

4.2 Messages Used in the Algorithm . . . . . . . . . . . . . . . 26

5.1 The Simulation Parameters . . . . . . . . . . . . . . . . 30

5.2 Comparison of RSS using PSO and TRS Algorithm . . . . . . . . . . . . . 46 


\section{List of Figures}

1.1 Density estimation of pick-up and drop activities for real Chicago data set for De-

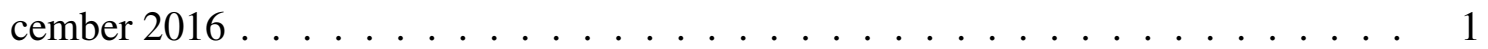

1.2 Dynamic Ride Sharing System: Three ride requests can be served by a single taxi (Source $(\mathrm{S})$, Destination $(\mathrm{D})) \ldots \ldots \ldots \ldots$

2.1 Standard flowchart of PSO [3] . . . . . . . . . . . . . . . . 6

2.2 Classification of Ride Sharing System [1] . . . . . . . . . . . . . . . . 7

2.3 Information passing in a Distributed System [4] . . . . . . . . . . . . . 10

2.4 The Architecture of the Real-Time Taxi-Sharing System [5] . . . . . . . . . . . . . 11

2.5 Taxi-Sharing Algorithm at Vehicle End [6] . . . . . . . . . . . . . . 13

3.1 Hourly Ride Request Distribution . . . . . . . . . . . . . . . . . . . 17

3.2 Analysis of Ride Requests Per Day in a Week . . . . . . . . . . . . . . . 18

3.3 Total trip requests, by hour of day, for a week [7] . . . . . . . . . . . . . 18

3.4 Average Trip Distance, by Hour of Day, for a Week . . . . . . . . . . . . . . . . . 19

3.5 Average Trip Duration, by Hour of Day, for a Week . . . . . . . . . . . . . . . . 19

3.6 Total Trip Requests, by Hour of Day, for a Week . . . . . . . . . . . . . . . . 20

3.7 Passenger Requests Variations in Summer (June 26 $6^{\text {th }}-$ July $2^{\text {nd }} 2017$ ) . . . . . 21

3.8 Passenger Requests Variations in Summer (July 10 $\left.10^{\text {th }}-16^{\text {th }} 2017\right) \ldots \ldots 22$

3.9 Passenger Requests Variations in Summer (July $17^{\text {th }}-23^{\text {rd }} 2017$ ) . . . . . . . . 22

3.10 Pick Up Requests Distribution around Chicago city . . . . . . . . . . . . . 23

4.1 High Level View of a Ride Sharing System . . . . . . . . . . . . . . . . . . . . . 24

4.2 Simulation Platform . . . . . . . . . . . . . . . . . . . 25

5.1 Global Best Values for a Combination of Particle Numbers and Epochs . . . . . . . 32

5.2 Overall Success Rate (OSR) of Ride Sharing Service (RSS) for Non-Peak Hours . . 34

5.3 Overall Success Rate (OSR) of Ride Sharing Service (RSS) for Peak Hours . . . . 35

5.4 Success Rate of $N^{t h}$ passengers (SNRP) on Weekdays . . . . . . . . . . . . . 35

5.5 Success Rate of $N^{t h}$ passengers (SNRP) on Weekends . . . . . . . . . . . . . . 35

5.6 Success Rate of $N^{t h}$ passengers on Sunday . . . . . . . . . . . . . . . . . 36

5.7 Distance Ratio (DR) for Non-Peak Hours . . . . . . . . . . . . . . . . 37

5.8 Distance Ratio (DR) for Peak Hours . . . . . . . . . . . . . . . . . . . . 37

5.9 Average Passenger Wait Time (AWT) in Seconds for Non-Peak Hours . . . . . . . 37

5.10 Average Passenger Wait Time (AWT) in Seconds for Peak Hours . . . . . . . . . 38 
5.11 Average Number of Messages Exchanged (ANM)for Non-Peak Hours . . . . . . 38

5.12 Average Number of Messages Exchanged (ANM) for Peak Hours . . . . . . . . . 38

5.13 Average Request Processing Time (ARPT) in Milliseconds for Non-Peak Hours . . 39

5.14 Average Request Processing Time (ARPT) in Milliseconds for Peak Hours . . . . . 39

5.15 Overall Success Rate (OSR) of Ride Sharing Service (RSS) for Non-Peak Hours . . 40

5.16 Overall Success Rate (OSR) of Ride Sharing Service (RSS) for Peak Hours . . . . 40

5.17 Success Rate of $N^{t h}$ passengers (SNRP) on Weekdays . . . . . . . . . . . . . 40

5.18 Success Rate of $N^{t h}$ passengers (SNRP) on Weekends . . . . . . . . . . . . . . 41

5.19 Success Rate of $N^{t h}$ passengers on Sunday . . . . . . . . . . . . . . . . . . . . . . . . . . . . .

5.20 Distance Ratio (DR) for Non-Peak Hours . . . . . . . . . . . . . . . . . . 41

5.21 Distance Ratio (DR) for Peak Hours . . . . . . . . . . . . . . . . . . . . . . 42

5.22 Average Passenger Wait Time (AWT) in Seconds for Non-Peak Hours . . . . . . . 42

5.23 Average Passenger Wait Time (AWT) in Seconds for Peak Hours . . . . . . . . . . 43

5.24 Average Number of Messages Exchanged (ANM) for Non-Peak Hours . . . . . . . 43

5.25 Average Number of Messages Exchanged (ANM) for Peak Hours . . . . . . . . . 43

5.26 Average Request Processing Time (ARPT) in Milliseconds for Non-Peak Hours . . 44

5.27 Average Request Processing Time (ARPT) in Milliseconds for Peak Hours . . . . . 44 
Dedication

Dedicated to my mom, dad and sister. 


\section{Acknowledgements}

I owe my gratitude to each of my committee members for providing me with constant support and guidance. I would like to thank my advisor Dr. Vaskar Raychoudhury for his guidance throughout my critical inquiry. It was a great journey working with such a patient and supportive mentor. He continuously revised my papers and provided feedback that encouraged me to organize the thesis well. Dr. Karen Davis rendered her valuable time and shared her topic expertise, thereby making this study more meaningful. I would like to thank Dr. Md Osman Gani for his expert suggestions and recommendations.

A heartfelt thanks goes to all my classmates, colleagues, and friends at The College of Engineering and Computing, Miami University.

Finally, my special thanks goes to Saurabh Kumar Karn, Urvashi Desai, Pallavi Timilsina, Naman Verma, Rina Harianto, and some special friends for making me believe that I can get through this and encouraging me with their kind words.

Thank you!! 


\section{Chapter 1 :Introduction}

\subsection{Motivation}

Proper functioning of modern society is largely dependent on an efficient transportation system. According to the National Household Travel Survey [8] in the United States, the average vehicle occupancy is approximately 1.6 and has not improved since 2009 . This is a common trend recognized in all major cities around the world such as Chicago, New York [9], San Francisco [10], Shanghai [11] and many more. As observed in the traffic index provided by the Chicago Data portal [12] the traffic congestion in Chicago has increased the travel time around 26\%, which equals an average of 29 minutes extra commuting time every day and can reach a maximum of 90 minutes delay. It is evident that the number of vehicles growing on the streets every day is adding to the congestion of the roads. A possible way to cut down the number would be through collective transport like ride-sharing which can match multiple riders to a common vehicle [13]. It also helps in making better utilization of the road, increasing the productivity of the drivers and saving energy [14]. The recent addition of autonomous vehicles promises increased safety on the roads, thus combining ride-sharing with an autonomous vehicle can boost the overall environmental benefits [14].

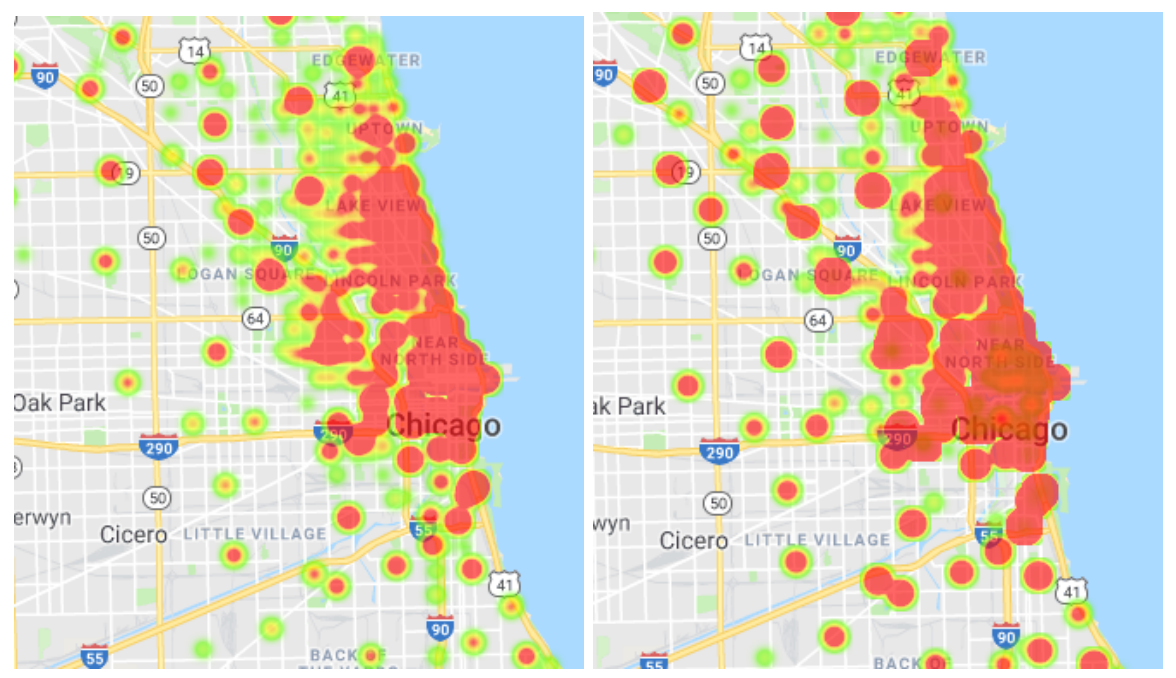

(a) Heatmap for pick up points

(b) Heatmap for drop off points

Figure 1.1: Density estimation of pick-up and drop activities for real Chicago data set for December 2016 
Fig 1.1a shows the city taxi pickup and Fig $1.1 \mathrm{~b}$ shows the city taxi drop-off locations in Chicago. From the images, it is apparent that most of the trips have originated from and culminated into the central Chicago region between River North and South Side shown by red shades. This area includes some of the major attractions of the city like Millennium Park, Navy Pier, museums and colleges. Due to such a high concentration of demands at the same location, ride-sharing provides a great alternative of transportation. Ride-sharing has developed in years and is a well liked researched topic these days. Dynamic Ride Sharing is a system that enables several passengers to share their taxi rides with fellow passengers in real time. Platforms like UberPool ${ }^{1}$, $L y f^{2}$ and $D i D i^{3}$ offer ride-sharing options which help the users to book a shared ride on the go through their smart devices. An example of dynamic ride-sharing can be seen in Fig 1.2, where three separate requests can be satisfied by a single taxi.

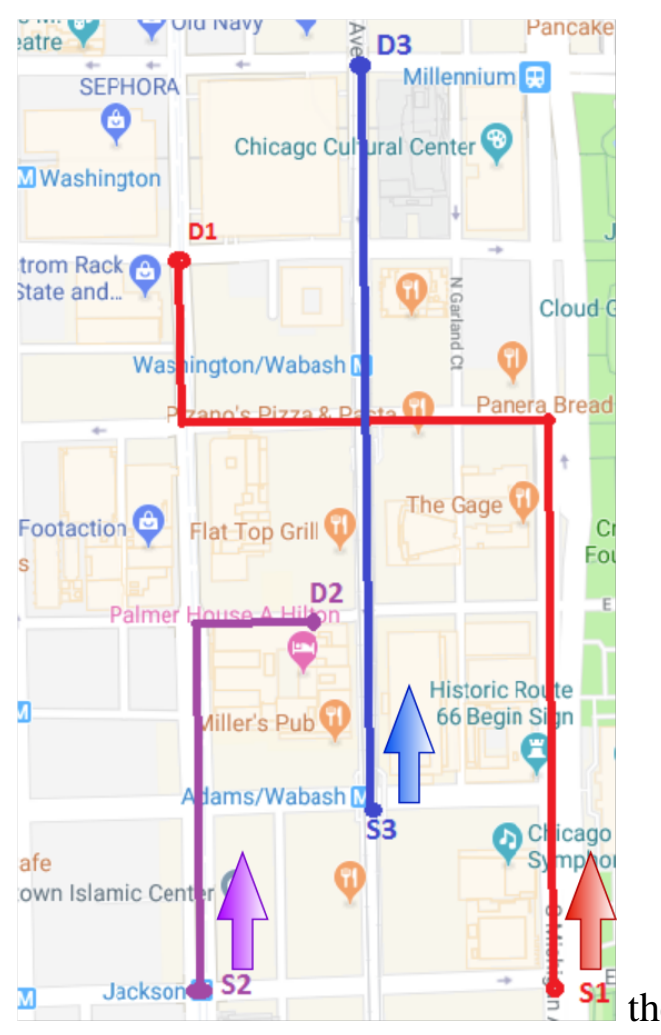

(a) Without Sharing

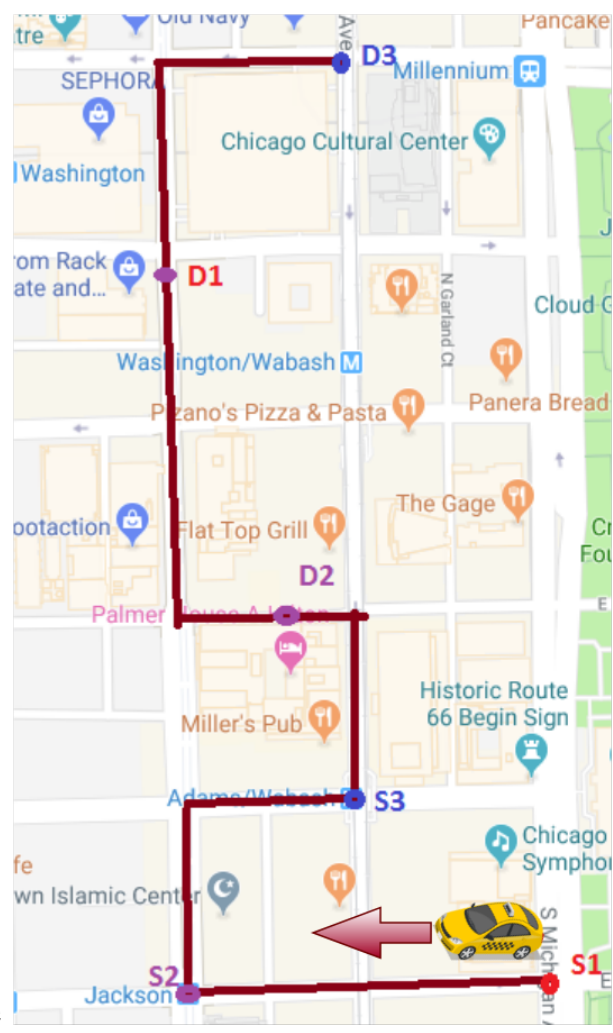

(b) With Sharing

Figure 1.2: Dynamic Ride Sharing System: Three ride requests can be served by a single taxi (Source (S), Destination (D))

The ride-sharing problem belongs to a classic Dial-a-Ride Problem (DARP) [15], which is considered an $N P$-hard problem. In DARP, the passengers request rides to travel from a specific origin(source) to specific destinations, which are fulfilled by either a single vehicle or multiple

\footnotetext{
${ }^{1}$ Uber: https://www.uber.com/

${ }^{2}$ Lyft: https://www.lyft.com/

${ }^{3}$ DiDi: https://www.didiglobal.com/
} 
vehicles. The combined goal is to find a minimum cost route for the vehicles to transport all the passengers. Owing to the non-deterministic nature of DARP, many heuristics, and non-heuristic algorithms are developed to solve it.

\subsection{Contributions}

In this thesis, a dynamic taxi ride-sharing system is designed to handle the incoming ride requests in real-time. This system provides flexibility for both the passengers and taxi drivers and will also be cost-effective for both. The contributions of the MS thesis include the following:

- Characterize prior research done in the field of ride-sharing

- Develop a dynamic ride-sharing system, capable of accepting and matching the ride requests in real-time extending the functionality of an existing algorithm

- Implement the Ride Sharing System (RSS) where passengers can select the taxi that fits their needs based on the cost and duration of the trip

- Create a test-bed dataset (including cleaning, extracting and transforming the data) from a large scale publicly available data

- Evaluate the system developed using a large scale real-world data of Chicago city

- Introduce additional metrics for performance evaluation and analysis

- Compare the performance of our RSS algorithm with previous algorithm designed by Batla et al. [11]

\subsection{Thesis Outline}

In this chapter, a brief introduction is provided to the research topic. It highlights the motivation for developing a dynamic ride-sharing system.

Chapter 2 provides a literature review of the significant work that has been done so far in the field of ride-sharing. It also contains a comparison with a related works and their challenges.

Chapter 3 highlights the details about the dataset, pre-processing and analysis of the dataset.

Chapter 4 highlights the proposed methodology and the algorithms designed for the RSS.

Chapter 5 includes the experimental setup and performance metrics used to evaluate the system designed. It also contains the results, validations, and the inference drawn from the results.

Chapter 6 concludes the work and lists possible future work in the field of dynamic ridesharing. 


\section{Chapter 2 : Background \& Related Work}

\subsection{Introduction to Optimization}

Optimization is a technique of finding workable solutions until no better solution can be found [16]. The solutions are accepted or rejected based on an objective. A significant number of studies consider single objectives, yet the problems in the physical world often command multiple objectives.

Multi-Objective Optimization (MOO) is related to mathematical problems which involve more than one objective function to be optimized simultaneously. This technique is generally used in the decision-making process, which has several criteria, and a decision is to be made based on those criteria. Most of the real world problems are based on MOO concepts. It is applicable in various fields of engineering and sciences. This model helps in the decision making process with some trade-offs.

Any given problem can be mathematically modelled using the MOO concept. The general form of MOO [16] problem is :

$$
\operatorname{Min} / \operatorname{Max} f_{m}(x), m=1,2,3, \ldots M
$$

Subject to,

$$
\begin{gathered}
g_{j}(x) \leq 0, j=1,2,3, \ldots J \\
h_{k}(x) \leq 0, k=1,2,3, \ldots K \\
x_{i}^{(L)} \leq x_{i} \leq x_{i}^{(U)}, i=1,2,3, \ldots, n \text { is the variable bounds. }
\end{gathered}
$$

As the name suggests, the problem has a number of objective function denoted by the function $f$ which can either be a maximize or minimize function. It is defined on some variable $x$, and $m$ denotes the number of objectives. These objective functions are subject to some constraints or trade-offs . In other words, these conditions needs to be full filled to reach the solution. A problem can either have no constraints or a number of constraints, for example an equality constraint $\left(g_{j}(x) \leq 0\right)$ and an inequality constraint $\left(h_{k}(x) \leq 0\right)$ from above equations.

The traditional way of solving MOO problems is the preference-based [16] approach, which attaches relative preference to the multiple objectives. However, the outcome of this technique was eventually a single optimized solution. Thus, non-traditional or stochastic search technique like evolutionary algorithms came into the picture. Evolutionary computation is a notion taken from the biological theory or nature for creating optimization methodology to solve problems using computers. Some examples of evolutionary approaches are Genetic Algorithms and Particle Swarm Optimization algorithms. 


\subsection{Particle Swarm Optimization (PSO)}

Particle Swarm Optimization (PSO) is an evolutionary computation approach developed by Kennedy and Eberhart [17] in 1995, inspired by social behavior of bird flocking and fish schooling. PSO was introduced for optimization of non-linear functions using the swarm theory. It shares some resemblance to other evolutionary computation techniques like Genetic Algorithm (GA) which involves evolutionary operations like mutation. PSO has been widely applied to many areas such as function optimization, fuzzy systems, artificial neural networks and many more. PSO is one of the many algorithms used to solve and optimize nonlinear functions. It does so by improving a candidate solution in an iterative manner until it reaches a given measure of quality. There is a considerable amount of research performed on solving the carpool or ride sharing problems using a variety of techniques, but evolutionary computational methods are not studied abundantly. When compared to other evolutionary optimization problems such as Genetic Algorithm, Ant Colony optimization and Simulated Annealing algorithm, PSO is simpler to implement, has a fast optimization speed and does not involve any complex mutation or crossovers. PSO is already successfully applied to a number of the nonlinear constrained optimization problem and was conceptualized to solve similar problems. Hence, a study in PSO will be helpful in exploring its application to the dynamic ride-sharing problem.

The PSO algorithm generally consists of these steps as shown in the Fig 2.1. In PSO potential solutions are initialized and are called particles and these are assigned to a random velocity. There each particle has a fitness value which is calculated using the objective function. The particles keeps track of it's the best solution (fitness) in the solution space called the pbest. Another best value known as the global best or gbest is also tracked, which is the best value obtained by the other particles in the system. This values are updated at each iteration and are directed towards a sufficiently good solution or till maximum number of iterations are reached. A maximum velocity $V_{\max }$, which is user-defined value, maintains a threshold on particle's velocities.

\subsection{Types of Ride Sharing Systems}

Ride-sharing problems are mostly modeled as optimization problems aiming to optimize one or more objectives and are usually solved as a mixed integer problem. Various heuristic and nonheuristic algorithms are used to solve the ride-sharing problem which can be broadly categorized into static and dynamic ride sharing [2]. Fig 2.2 shows the classification of the ride-sharing systems. The dynamic ride-sharing is further divided into three categories, centralized, distributed and hybrid. As seen in in the survey done by Silwal, Gani and Raychoudhury[1], the following sections explore all the variations in details.

\subsubsection{Static Ride Sharing}

In static ride-sharing, the number of riders as well as the route is known in advance. It is usually seen as a carpool service where a set of passengers board the vehicle together at a common origin and travel together until a single common destination. Passengers may also de-board at any intermediate locations. In these scenarios, the origin and destination of every rider are communicated 


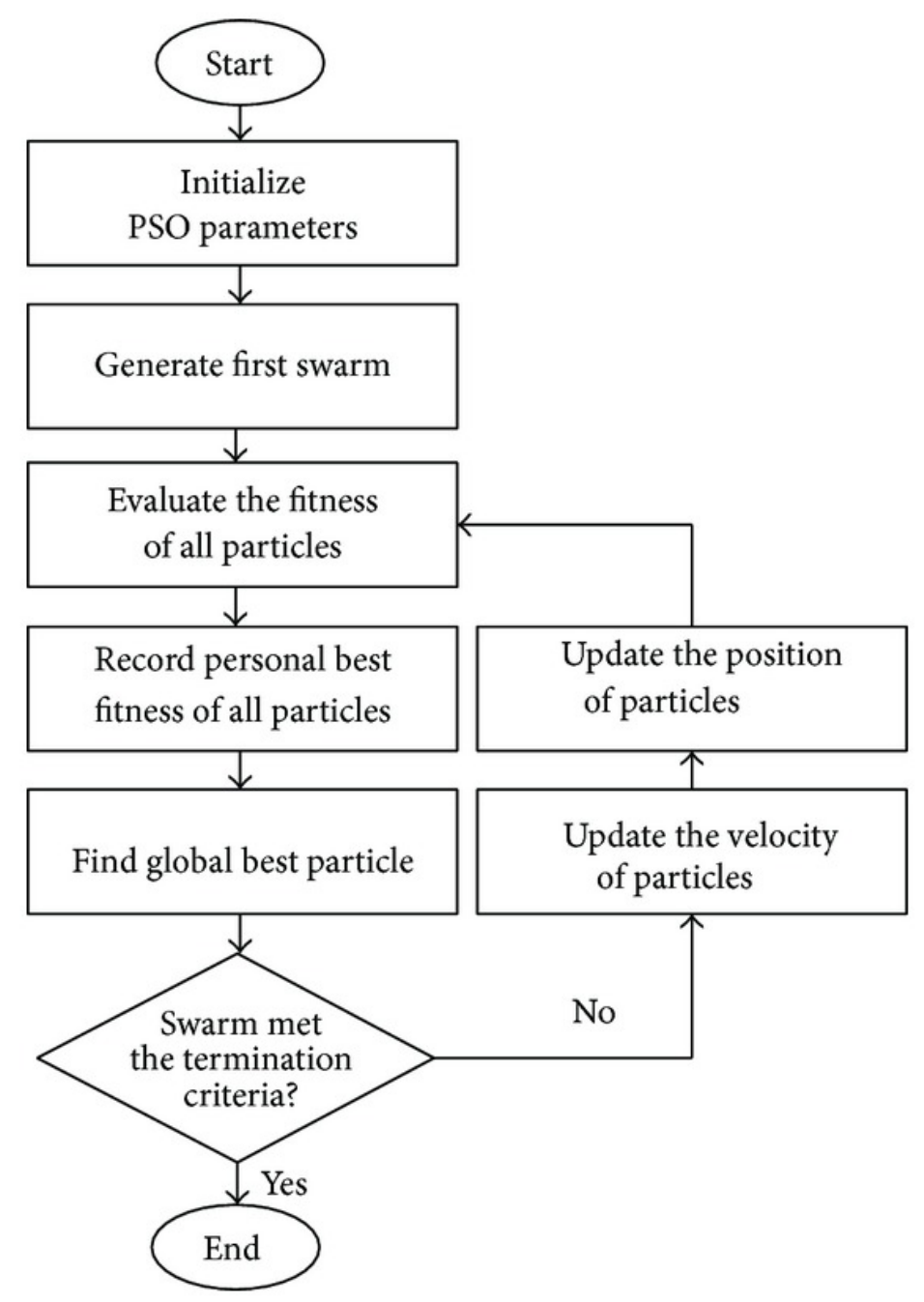

Figure 2.1: Standard flowchart of PSO [3]

earlier and subsequently, a feasible final route is decided.

For instance, Psaraftis [15] formed the static ride sharing problem using a general objective function to minimize the total time to transport all the passengers. He introduced an exact approach using dynamic programming which performed significantly better than the Travelling Salesman Problem (TSP) of similar size.

Baldacci, Maniezzo, and Mingozzi [18] proposed both heuristic and non-heuristic approaches for static ride-sharing in the form of carpooling. Their technique was based on two integer programming formulations based on Lagrangian Column Generation. The path generation was done using dynamic programming methods. Ma et al. [19] presented a similar non-heuristic solution using a linear algorithm.

Jean-Francois Cordeau [20] introduced the ride-sharing problem as a mixed-integer program- 


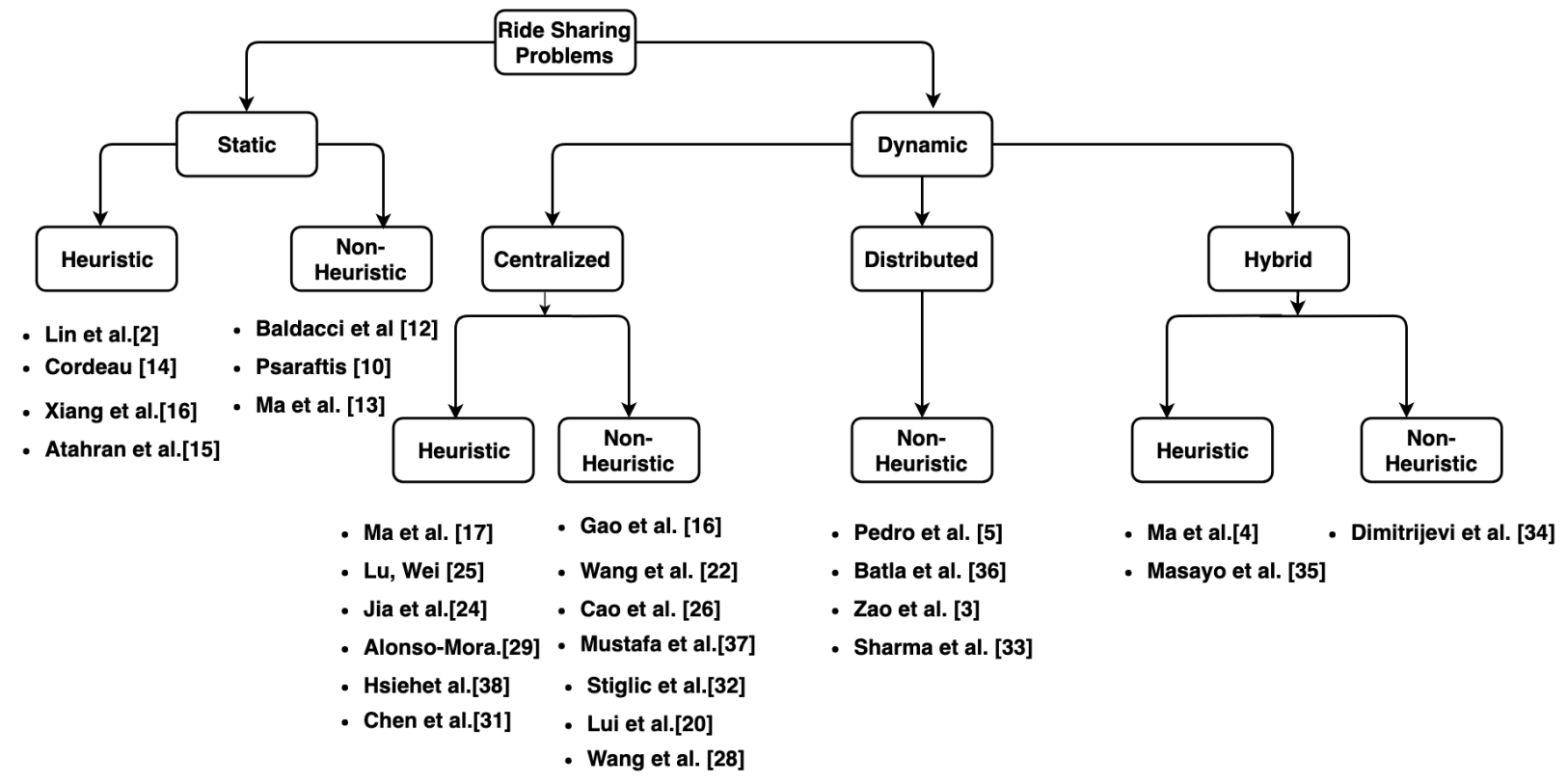

Figure 2.2: Classification of Ride Sharing System [1]

ming format and the branch-and-cut algorithm. His approach was a combination of heuristic and non-heuristic methods. He explored a number of constraints or 'valid inequalities', for example, capacity, duration, time window, pairing, precedence, and ride-time constraints. The approach was successfully applied to small or medium-sized instances, so he claimed, it would work well to optimize small subsets of the larger problem. He formulated the problem as below:

$$
\operatorname{Min} \sum_{k \in K} \sum_{i \in N} \sum_{j \in N} c_{i j}^{k} x_{i j}^{k}
$$

The objective function 2.4 is the minimization of the total routing cost subject to a large number of constraints.

In the same manner, Atahran et al. [21] proposed a multi-criteria ride-sharing problem based on Cordeau [20], that uses a genetic algorithm to obtain Pareto Optimal solution. Xiang, Chu, and Chen [22] also presented a heuristic technique for static ride sharing using organized local search algorithm. Lin et al. [23] developed a routing optimization model for a static ride-sharing problem in terms of the travel mileage, waiting time and additional travel time due to ride-sharing. They offered a probabilistic approach using simulated annealing algorithm and were able to save $19 \%$ mileage for a network having 9 nodes.

Such static solutions were successful for certain types of ridesharing solutions but do not work well in the absence of the static information available before decision making. 


\subsubsection{Dynamic Ride Sharing}

Dynamic ride-sharing makes no assumptions about the knowledge of the number of riders and their origin and destination and the number of available vehicles. The requests should be handled in short notice or on the fly and as and when they arrive (run-time adaptation). Dynamic ridesharing problems are also modeled as optimization problems.

Ma et al. [24] divided the problem into two parts, first a searching algorithm to find prospective taxi using spatiotemporal index while the other half was a scheduling algorithm. The road networks were partitioned into grids and simulation was done on a huge GPS trajectory data. The results indicated an improvement in the number of shared-riders with less travel distance. Similarly, Gao et al. [25] also defined the problem in two parts, the first one being taxi scheduling which is achieved using binary search and the second part is defined as the taxi scheduling which tries to optimize the system. Similar, approaches were presented by Archetti et al. [26], but a special case of drivers that participate in ride-sharing occasionally was captured.

Liu et al. [27] proposed a branch-and-cut algorithm for a variation of DARP, which eliminates unnecessary variables and constraints and narrows the solution space. Similarly, Agatz et al. [28] provided some pre-processing techniques and introduced a Rolling Horizon Approach(RHA), in which the riders and taxis could freely enter and leave the system. Wang et al. [29] proposed a stable or nearly-stable ride-sharing matches using the RHA discussed above. The paper also highlights the system degradation due to the refusal of matches.

In their paper, Zhu et al. [30] define a dynamic solution for the public vehicle (PV) system which depends on a centralized cloud system for handling passenger requests. Instead of using exhaustive search techniques generally used which can be tedious and inefficient, their algorithm focuses on reducing the global search to local search. Although there might be a trade-off between the PVs and passengers goals, the algorithm tries to attain the best social welfare for both.

Jia et al. [31] viewed the ride-sharing requests and demands as an imbalanced matching problem and tackled it using a dynamic algorithm. They tried to solve the problem in parts by designing approximation algorithms using Greedy approach for a static problem and other heuristic algorithms for both static and dynamic environment. The optimization is done using the greedy method and adjusted to the Maximum node-disjoint paths model. Similar to the previous paper, Zhu et al. [30] proposed a model that focuses on maximizing the drivers total profits and maximizing social welfare. In his thesis, Lu [32] compared a large-scale ridesharing problem with Travelling Salesman Problem (TSP) and the vehicle routing problem (VRP). A mixed-integer program (MIP) model and an Insertion-based heuristic approach are used. However, the MIP model does not guarantee to solve larger instances efficiently as the problem is defined as an NP-Hard problem.

\subsection{Dynamic Ride Sharing Architecture}

The dynamic approach provides more flexibility and effectiveness compared to the conventional or static ride-sharing method. The system architecture for static ride-sharing is a central system that is provided with all the ride requests and vehicles. It then creates optimal routes with

minimum cost for the system provider. However, the dynamic ridesharing systems can be designed 
in a non-centralized or distributed and as hybrid systems. These system architectures are explained in details in the following section.

\subsubsection{Centralized Ride Sharing}

A centralized ridesharing system consists of a central server that is responsible for making all the request and ride matches. Such systems will receive requests centrally and are aware of the number of taxicabs currently in the system and also their spatial coordinates. However, due to dependency on a single machine or instance, this central system is susceptive to failure and does not adapt well with the increasing numbers of participants (passengers and taxis).

The dynamic ridesharing problem can also be considered as a ride matching problem. A unique approach to such matching is proposed by Cao et al. [33]. They proposed SHAREK; a scalable and efficient ride-sharing service which employs early pruning techniques to reduce the computational complexities for calculating the shortest path. The drivers register themselves in SHAREK when they are ready to provide services to the passengers. On receiving the ride requests, SHAREK matches drivers to the requests based on a cost model. They divide the pruning method into three phases. While the first two phases prune requests based on time and cost considering Euclidean distance, the final phase prunes for shortest distance using the Semi-Euclidean distance before fetching the actual road network route. The system relies heavily on a matching table which stores candidate drivers from each phase and is carried forward. Such tables do add limitations when the size of the ride requests and the number of registered taxis increase significantly. They claim that the early pruning techniques improve the efficiency and scalability as the elements that do not belong to a potential solution is removed in advance. An extension to SHAREK [33] was proposed by Ning et al. [34] using heuristics for large-scale road networks having a number of turns and one-way roads.

Wang et al. [35] proposed a centralized ride-sharing framework. In this framework when a new booking request is made, it goes through the share trip plan formation process. Here, an active booking request which is not yet served is matched with the incoming request and then the share plan is inserted into the trip queue $(Q)$ which is assigned to a free taxi. Their system defines a number of conditions, for instance, time constraints (departure and arrival), capacity constraints and taxi fare constraints. Instead of focusing on optimization, they took a greedy approach to solve the problem, which chooses the locally best booking and does not bother about the globally optimal shared trip plan. They claim that this approach helps to get a shorter response time and reduces the computational complexity of the problem. Alonso-Mora et al. [36] follows a similar method and in addition balances the vehicles from non-busy areas to areas of higher demand. Berbeglia et al. [37] proposed a centralized ride-sharing scheme which is based on Cordeu [20] but applied for a dynamic setting.

In their paper, Chen et al. [38] proposed an event-driven system in order to incorporate a more real-time nature to its implementation and results are obtained using city maps and real taxi traffic data. The proposed to use Receding Horizon Control approach (RHC) to solve the ride-sharing optimization problem over a given planning horizon and it takes advantage of the event-driven nature. Similarly, Stiglic et al. [39] proposed a system that also included public transport. 


\subsubsection{Distributed Ride Sharing}

A distributed RSS, does not have a dependency on a central system. This paradigm utilizes a combination of entities between which the computation is divided and they communicate through message passing. Thus, the system can grow substantially without any compromise in performance. This type of RSS is highly dependant on the underlying network layer and can crash due to network failure.

Zao et al. [4] designed a Vehicle Ad Hoc Network (VANET) model that included vehicles with high-quality sensor nodes. A Social Group Architecture (SGA) was introduced by them. Their framework relies on peer-to-peer communication and scattered communication among participating entities named as vehicle to infrastructure communication (V2I) and vehicle to vehicle communication $(\mathrm{V} 2 \mathrm{~V})$ as shown in Fig 2.3. They establish a social group as a graph of all the members involved as nodes and a correlation between the members as an edge. The ride requests are transmitted to the relay vehicle until a match is found. The VANET model reduces the heavy reliance on the network and is claimed to be a robust system. However, all the devices in this architecture should have support for the $802.11 \mathrm{p}$ protocol to add wireless access in vehicular environments.

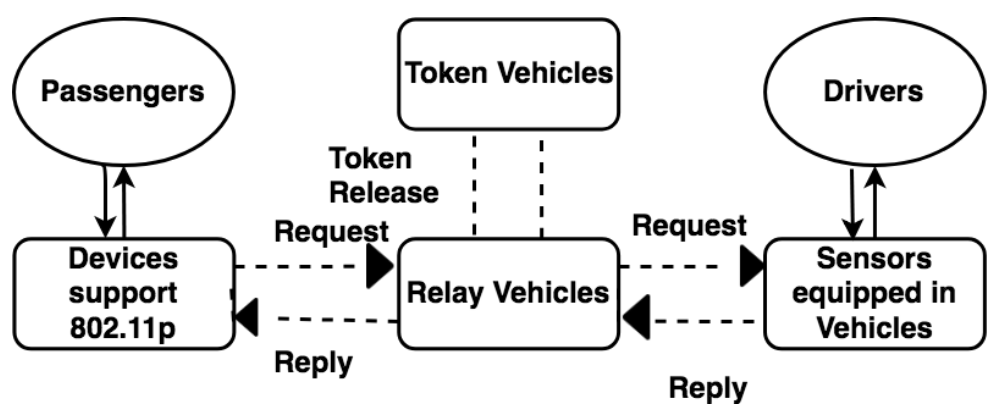

Figure 2.3: Information passing in a Distributed System [4]

Following a similar approach, Sharma, Seo, and Jong [40] proposed network architecture for vehicles based on blockchain (Block-VN) in a smart city. They propose Block-VN as a secure and distributed technique for this pattern which is dependant on networks-and-vehicle interactions. However, it is crucial for the vehicles to be connected to the blockchain vehicular network and have other configurations (sensors, data storage, computing resources) capabilities as well.

In their recent paper Yu, Raychoudhury and Silwal [7] proposed a purely distributed ridesharing algorithm using localized communication between the passengers and taxis. They have chosen two day data (07-26-2017 and 07-27-2017) and have used those day's data for exeperimentation. Their non-heuristic route validation algorithm ensures a $76 \%$ success in ride-sharing. They extensively tested these data for a wireless transmission range of 50, 100, 200, 250 and 500 (metres).

\subsubsection{Hybrid Ride Sharing}

A hybrid RSS aims to combine the positive aspects of both the central and distributed models. The distributed feature is achieved using a cluster of servers or virtual machines. In this model, the 
load is distributed through a distributed cloud environment or through parallel computation. The distributed central core handles complex computations and is always connected through infrastructural network environment. These types of systems are quickly gaining popularity and provide a practical solution to the huge demand in ride-sharing space. Such a system is easy to scale and an addition of preventive measures can makes the hybrid ride-sharing system fault tolerant.

Dimitrijević et al. [41] recognized a need to develop a model having a global reach in realtime. Many systems developed before were limited to a certain number of mobile clients and was incapable of handling a growing number of clients. They proposed to develop a system which can grow seamlessly using cloud distribution strategies. Thus renting out cloud resources for horizontally scaling the ride-sharing system is implemented along with data scaling using non-relational databases. This model can be pictured as a separate driver and a passenger client making requests to their respective server nodes hosted in the cloud. A messaging queue caches the requests and responses. It also stores the information in various database instances in the cloud. They claimed that they were successful in creating a prototype and testing it using a handful of users as both passengers and taxis.

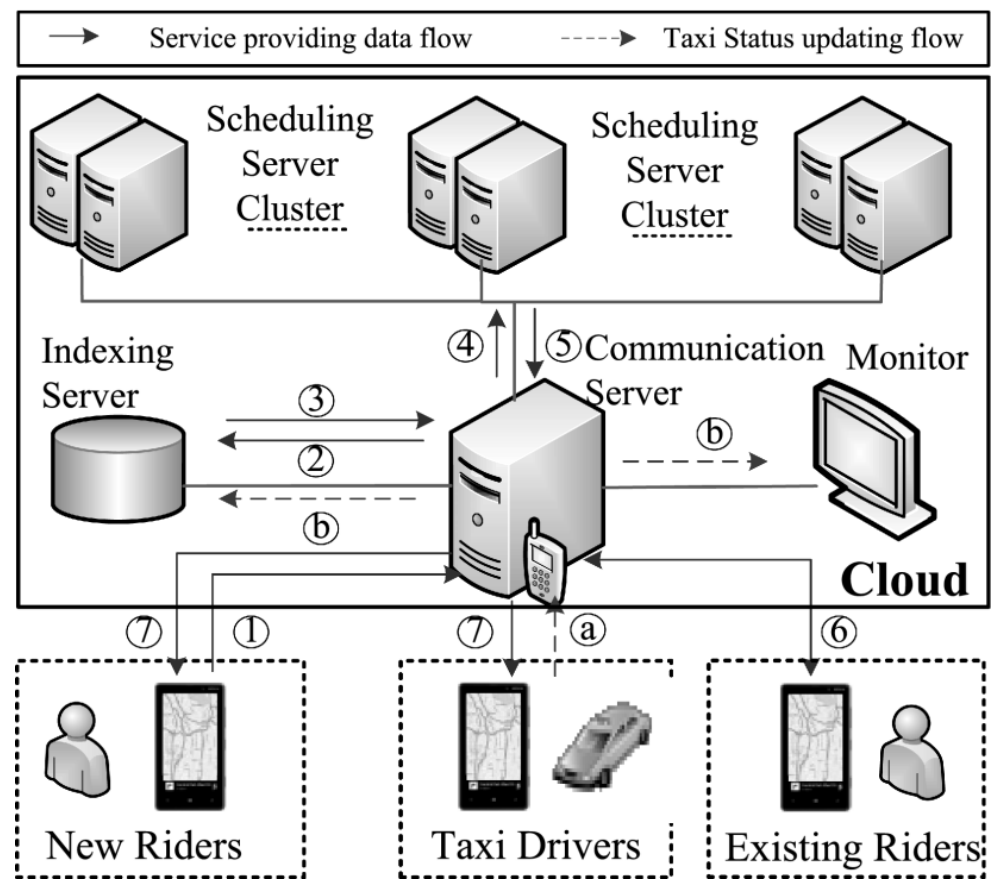

Figure 2.4: The Architecture of the Real-Time Taxi-Sharing System [5]

Ma et al. [5] designed a real-time dynamic ridesharing system using mobile cloud architecture. Fig 2.4 shows the architecture of the real-time system. They define a cloud having a number of servers and a monitor that keeps track of their running status. Similar applications with separate interfaces are provided to taxis and riders. A distributed cloud system is easy to scale, but for the implementation purposes, they have simulated the cluster using indexing server and scheduling servers as seen in the figure. They suggested the system can be improved using clustering techniques like Windows Azure A2 virtual machines. While their system mimics a clustered ar- 
Table 2.1: Pros and Cons of different RSS architecture [1]

\begin{tabular}{|l|l|l|}
\hline \multicolumn{1}{|c|}{ Type } & \multicolumn{1}{c|}{ Advantage } & \multicolumn{1}{c|}{ Disadvantage } \\
\hline \multirow{2}{*}{ Static } & Simple applications & Non-scalable \\
\cline { 2 - 3 } & Optimal for smaller instances & Not applicable to real-time ride-sharing \\
\hline Dynamic & No prior scheduling required & Shorter response time makes the system development complex \\
\hline Centralized & A single system performs all the functions & Non-scalable \\
\hline \multirow{2}{*}{ Distributed } & Does not rely on a central entity for decision making & Hardware dependent \\
\cline { 2 - 3 } & Highly scalable & Requires supporting network \\
\cline { 2 - 3 } \multirow{3}{*}{ Hybrid } & Decision making is distributed among all the participating components & \multirow{2}{*}{ Dependent on an internet connection } \\
\cline { 2 - 3 } & Provides load sharing & \\
\cline { 2 - 3 } & &
\end{tabular}

chitecture, it is still a simulation found in a single machine, thus any failure in the single machine would crash the entire system.

Masayo et al. [42] proposed a centralized model which has a passenger fleet and taxi fleet along with the simulator. They assert that the taxi and the passenger systems work independently due to parallelism. Their system is implemented using Hadoop's MapReduce which helps in the analysis of the data. They make use of heuristics to find the best route. Similar to the earlier approach [5], they use indexing scheme combined with parallelism to scale the simulator effectively.

In summary, the identified advantages and disadvantages associated with all the afore-mentioned types of ride-sharing systems are listed in Table 3.1. By listing the strengths and weaknesses of each type of systems, we can decide on the most desirable design decisions and possibly improve the performance of the RSS.

\subsection{Related Work}

This section consists of similar work done in the field ride-sharing. The different optimization models and various distributed and dynamic ridesharing systems. A brief discussion of the differences and similarities in the approaches is presented.

The features, constraints, and objectives of a static and dynamic ride-sharing problem were highlighted in details by Agatz et al. [2]. Their aim was to provide a better direction for various optimization approaches that were similar but not limited to the transportation-related problems. They briefly highlighted the dynamic addition of drivers and riders in the system. The expectation of unknown future requests by the taxis and re-arrangement of pre-scheduled trips. They list four variants of the ride-sharing system as shown in the Table 2.2. These variants are based on the different ways of matching the drivers and riders. The most prevalent type of dynamic ride-sharing based on smart devices involve multiple drivers and riders. In such systems, making suitable matches as well as deciding on optimal routes becomes complex. This paper serves as a fundamental guide to a dynamic ride-sharing problem as it explores the challenges for developing a dynamic system. Although their study does not include an implementation of the dynamic paradigm in details, it leads towards the solution.

In their paper [6], d'Orey et al. proposed a novel dynamic and distributed taxi-sharing system. Their goal was to design a flexible system for both passengers and taxis which was cost effective. They assert that by making the system distributed, i.e, performing the complicated computations in each vehicle level rather than at a central location, a smaller processing time is possible to achieve. 
Table 2.2: Ride-Share Variants [2]

\begin{tabular}{|l|l|l|}
\hline & Single Rider & Multiple Riders \\
\hline Single Taxi & Matching of pairs of taxis and riders & Routing of taxis to pickup and deliver riders \\
\hline Multiple Taxis & Routing of riders to transfer between drivers & Routing of riders and drivers \\
\hline
\end{tabular}

Fig 2.5 shows the distributed algorithm at the taxi/vehicle side. The taxi and passengers make use of wireless technology to send and receive requests and responses. On receiving a new request, a permutation of all the routes are computed and filtered based on the constraints. The cost of the distance is calculated based on the shortest path. The maximum service degradation factor, that is the additional distance due to ridesharing also needs to be considered. The passengers receive the cost estimation when all the constraints are met. The algorithm at the passenger side can select the response having minimum cost. The goal of the proposed work is to develop a similar distributed and dynamic model proposed by them. The simulated system was tested with a small number of instances of taxis and passengers. The proposed work plans to define proper algorithms at both ends and implement the system using a large scale real data set.

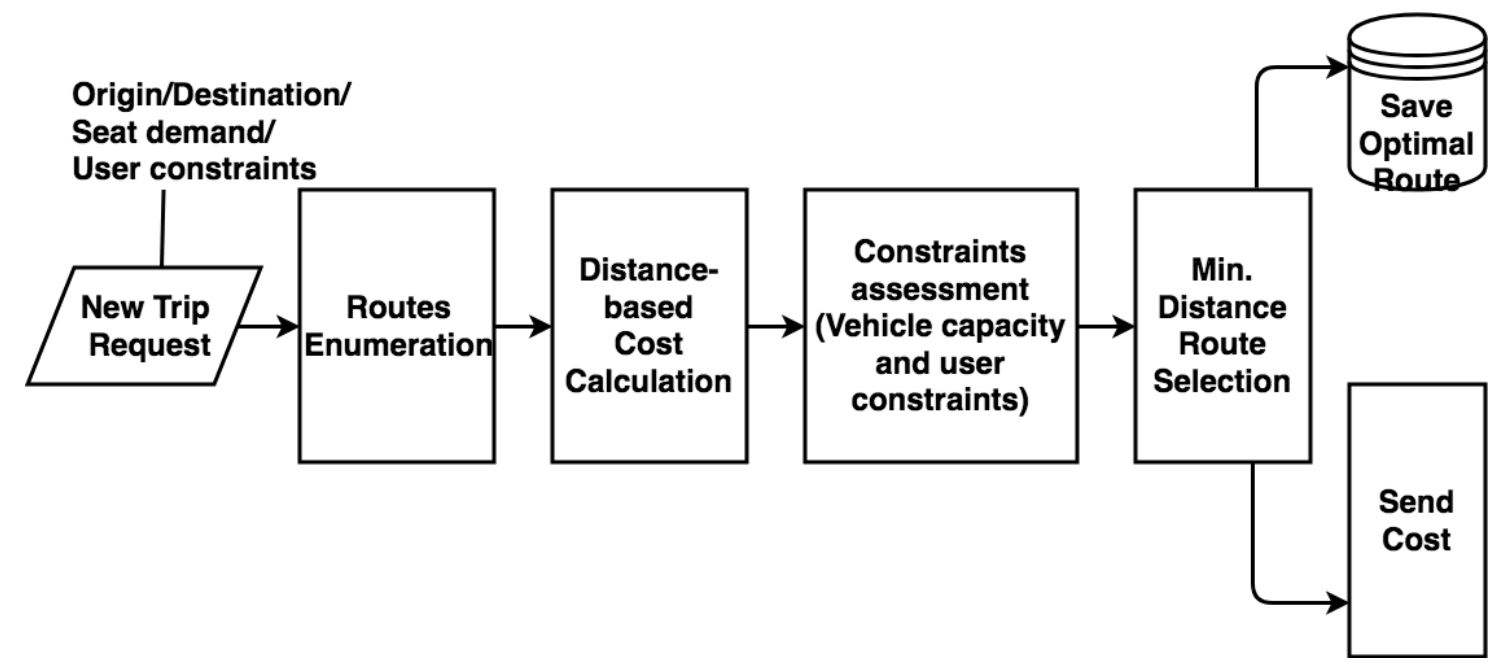

Figure 2.5: Taxi-Sharing Algorithm at Vehicle End [6]

Batla et al. [11] proposed a similar Taxi Ride Sharing (TRS) algorithm as d'Orey et al. [6] that divides the problem into searching and scheduling of rides. They proposed a distributed algorithm for ridesharing that used localized communication between passengers and nearby taxi drivers. It can handle real-time ride requests without disturbing the confirmed passenger trip schedules. They have tested the algorithm with a large scale GPS traces of 4,000 taxis in Shanghai, China. As the taxi traces are of single passenger rides, they have applied clustering (DBSCAN) to spatially combine the requests in order to simulate parallel ride share requests. Their evaluation of the system is based on improvement in drivers profit and passenger's cost savings while reducing the total distance traveled (while considered without ride-sharing). This paper has similar goals to the proposed study such as an increase in shared rides for the taxis, as well as cost-effectiveness for both taxis and passengers. 
Lokhandwala and Cai [43] proposed an agent-based model having a matching algorithm between taxi and passengers. They modeled using traditional taxis as well as autonomous vehicles including the complexities of real-world scenarios and road infrastructures. They included a number of added preferences such as maximum deviation allowed, sharing participation level and also modeled the changing shifts in traditional taxis. This type of taxi shift change is applicable for traditional taxis and does not fit in the modern application based ride-sharing. Their RSS is evaluated based on fleet reduction, which is reducing the total number of the taxi, by increasing the resource (the taxi space) utilization. Improvement is noticed which adds to the environmental benefits as well. They claim that the system was tested up to 8000 fleets (taxi) using New York-TLC data set size but do not mention how they can handle fleet size above that limit. Extensive analysis of the results is performed with the help of different types of graphs and charts, which provides a very clear interpretation of the results. A similar analysis can be included in the proposed work, for better visualization and conclusion.

Hsieh et al. [44] approached the dynamic ridesharing problem using double auctions, and constructed the problem as an integer programming problem. Their approach is very close to the proposed work, as the problem is framed in a similar way using evolutionary (meta-heuristic) technique. The objective function is defined as the overall cost savings as seen in Equation 2.5. subject to few constraints. The overall cost savings is obtained by adding the real bid price, cost of the driver traveling alone and subtracting the cost of transporting the passengers. However, the proposed system will include a separate objective for both passengers and taxis as well as a common objective for the ride-sharing system. Since, its' a bidding system, the cost function depends on the initial price bids, the cost of a driver traveling alone and the cost of transporting the passengers. They devised the problem to maximize the cost savings keeping in mind the consequences of adding multiple rides in a single vehicle can result in a detour delay. They developed a discrete coevolving particle swarm optimization (DCCPSO) algorithm, a meta-heuristic approach to solving the single objective function. They test the system using small numbers of taxis and passengers. Their study claims that DCCPSO performs significantly better than the other existing algorithms for solving carpooling problems.

$$
F(x, y)=\Omega_{1} \sum_{p=1}^{P} y_{p}\left(f_{p}\right)+\sum_{d=1}^{D} \sum_{j=1}^{J d} x_{d j} o_{d j}-\sum_{d=1}^{D} \sum_{j=1}^{J} d x_{d j} c_{d j}
$$

\subsection{Conclusion}

Table 2.3 provides a summary of the features of the related work reviewed in the previous section. We have chosen only a dynamic approach to ride-sharing for comparison. This table also lists out the heuristic and non-heuristic approach the authors have taken in their papers. The distributed or centralized design the authors have taken for performing experiments. Some authors have chosen to combine both or in other terms a hybrid design. It also lists helps to understand the size of the dataset (small or large scale) being used for running experiments.

We designed a dynamic ride-sharing system (RSS) which primarily uses a heuristic algorithm for ride-matching. The heuristic algorithm selected is the Particle Swarm Optimization due to 
Table 2.3: Related Work Comparison

\begin{tabular}{|l|c|c|c|c|c|}
\hline Author(s) & Agatz et al. [2] & d'Orey et al.[6] & Batla et al.[11] & Lokhandwala and Cai [43] & Hsieh et al.[44] \\
\hline Dynamic Technique & Defined(Not Implemented) & Implemented & Implemented & Implemented & Implemented \\
\hline Non-Heuristic & Yes & Yes & Yes & Yes & - \\
\hline Heuristic & - & - & - & - & Yes \\
\hline Distributed Design & - & Yes & Yes & Yes & Yes \\
\hline Centralized Design & Yes & - & - & Yes & Yes \\
\hline Applied To & - & Small Scale Data & Large Scale Data & Large Scale Data & Small Scale Data \\
\hline
\end{tabular}

its fast optimization speed as discussed in the background. It also uses secondary non-heuristic algorithms which are an extension of previous works. The system will leverage the benefits of both distributed and centralized design. A resilient RSS design to handle large scale data is designed and implemented for the thesis work. 


\section{Chapter 3 : Data Set and Pre-Processing}

Chicago is situated beside Lake Michigan in Illinois and is one of the largest cities in the U.S estimated to have a population of around 2.7 million in 2016 [45]. The taxi trips data set from the Chicago Data Portal ${ }^{1}$ is taken for doing our experiments. The dataset is a comma-separated values (CSV) file of approximately $43 \mathrm{~Gb}$. During our experimentation, it had the data from 2013 through August 2017. The dataset consists of 24 columns, and the total number of records is 112,860,054. However, the columns with null values or zero values for total miles, total seconds, pick up, drop off latitude and longitude are removed. So, the final number of records was reduced to $93,285,184$. Due to a large number of records, the dataset could not be viewed using Excel sheets or Notepad++ editor. The analysis and pre-processing were done using Python (version 3). The dataset has now been updated up to October 1, 2019. Some of the relevant columns (the columns of data which we have used while conducting our experiments) and their descriptions are presented in Table 3.1.

Table 3.1: Few columns from the Dataset

\begin{tabular}{cc}
\hline Columns & Description \\
\hline Trip ID & A unique identifier for the trip \\
Taxi ID & A unique identifier for the taxi \\
Trip Start Timestamp & Date-Time recording the beginning of the trip \\
Trip End Timestamp & Date-Time recording the end of the trip \\
Trip Seconds & Time of the trip in seconds. \\
Trip Miles & Distance of the trip in miles. \\
Fare & The fare for the trip. \\
Pickup Centroid Latitude & Latitude of the pickup \\
Pickup Centroid Longitude & Longitude of the pickup \\
Dropoff Centroid Latitude & Latitude of the dropoff \\
Dropoff Centroid Longitude & Longitude of the dropoff \\
\hline
\end{tabular}

\subsection{Data Pre-processing}

In order to protect the privacy of individuals, some columns in the data set are an approximation of the actual value rather than the actual values. For example, the taxi id and the trip id are not the actual ids but rather specifically created for this data set. Also the Trip Start Timestamp and Trip End Timestamp are rounded to the nearest 15 minutes and then published as shown in Fig 3.1a. However, in order to use the given data, the pickup time had to be randomized to make it closer to

\footnotetext{
${ }^{1}$ Chicago Data Portal: https://data.cityofchicago.org/Transportation/Taxi-Trips/wrvz-psew
} 
the real world scenario. An hour data is initially grouped into four categories of 15 minutes delta ie. at $0,15,30$ and 45 minutes time stamp. So to uniformly distribute the time, the group of records at the 0th minute is picked and for each entry in that category, a time between 0th to 15th minute is randomly assigned. Similar idea is applied for other minute groups as well. Finally, a uniform distribution of time is obtained within that hour as shown in Fig 3.1b.

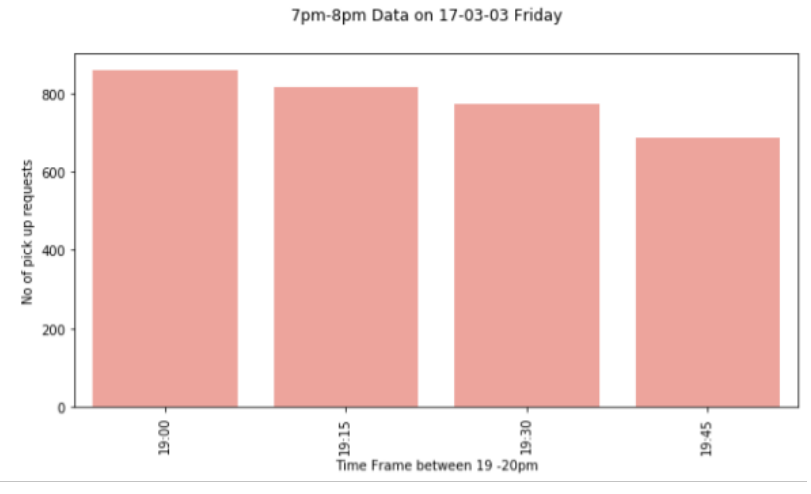

Fig 1 . Hourly ride request distribution without randomization

(a) Without Randomization

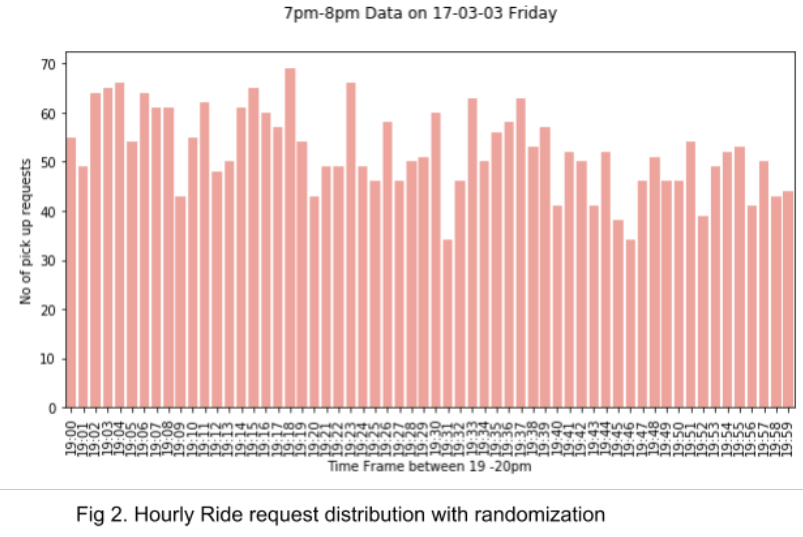

(b) With Randomization

Figure 3.1: Hourly Ride Request Distribution

\subsection{Data Analysis}

The average number of requests per day in a week is shown in Fig 3.2. It can be observed from the graph that the highest number of requests are made on weekends, Friday has the maximum requests compared to Saturday and Sunday. As Friday is the beginning of weekends, most people make plans to travel away from home or workplace and travel to those places.

Similarly, the total number of requests were compared hourly for each day in a week as seen below. It can be seen the maximum number of pick up requests are recorded on Friday evening. From the Fig 3.3, it is noticeable that the maximum requests are made after midnight until early morning on Saturday and Sunday. Since people are mostly out until mid-nights on Fridays they tend to return after midnight which falls on Saturday. The requests gradually decrease after evening hours until midnight for all the days except for Friday and Saturday, which explains the weekend behavior.

The graph shown below in Figure 3.4 helps to visualize the average distance of the rides per hour per day of the week. The average distance traveled during Monday morning is little over 6 miles. These distance range from 35 miles to as low as 0.5 miles, thus we obtain such an average. The longer distances are recorded for people traveling from airports to their homes or offices. Since people want to reach office on time they start very early in the morning, thus we see a spike in the early hours. The longest distance of the ride is on Mondays, probably most people trying to reach their workplaces. It is noticeable that the pattern is very similar for most days, except for Monday. 


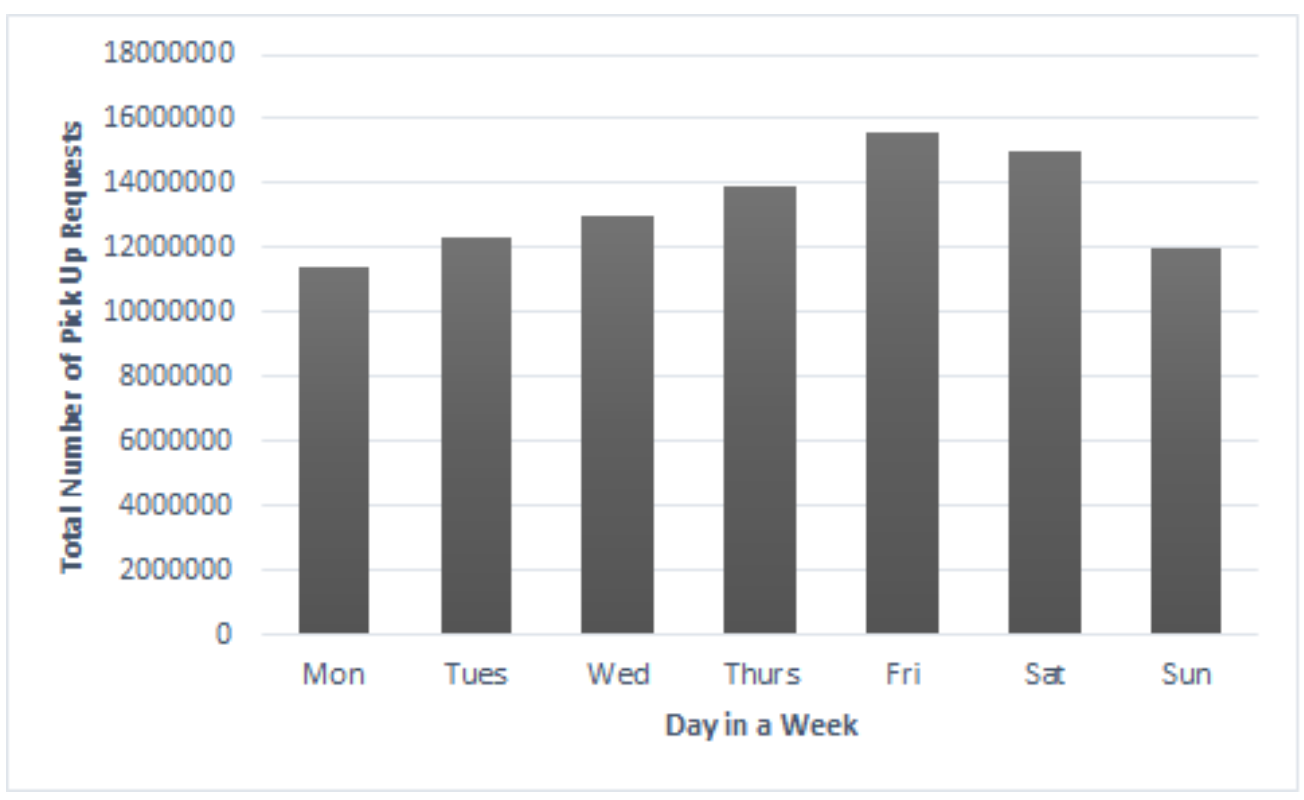

Figure 3.2: Analysis of Ride Requests Per Day in a Week

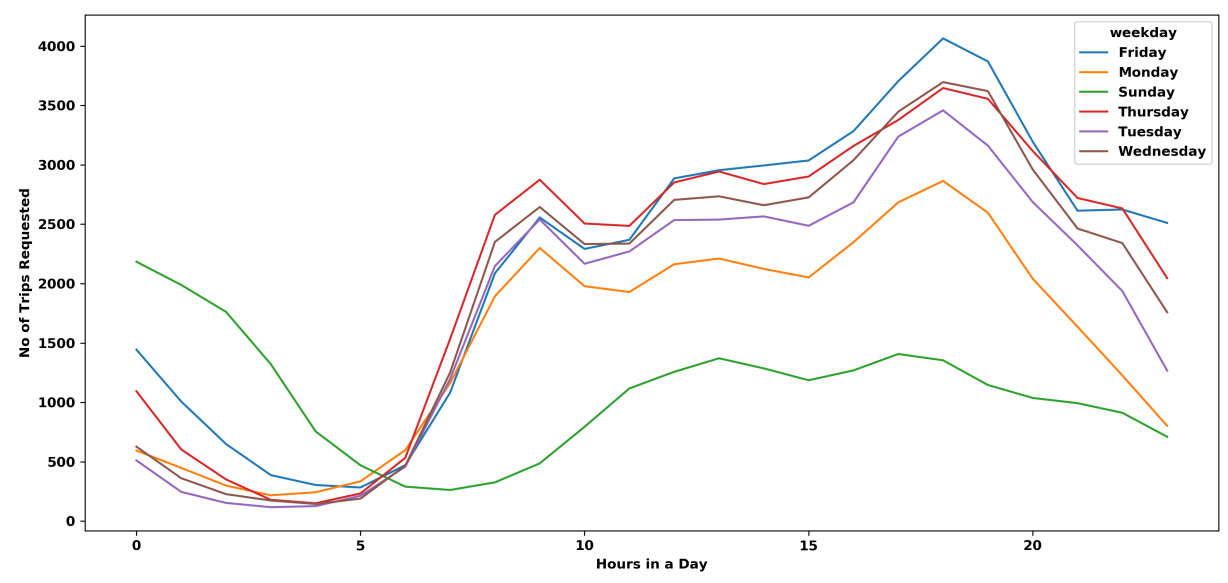

Figure 3.3: Total trip requests, by hour of day, for a week [7]

The average duration of the rides throughout the day in a week can be envisioned in Fig 3.5. The longest duration of the ride is on Mondays, probably most people trying to reach to work. It is noteworthy that the pattern is different on a Monday. People take rides for shorter duration at night starting from $8 \mathrm{pm}$ till early mornings. We can see two peaks in the values that correspond to office hours in the morning and end of office hours in the evening. So the ride duration are longer during the morning hours starting from 5 am till 10 am which decreases steadily till noon and again catches up the afternoon.The peak value is 1100 seconds ( 18 minutes approx) during 


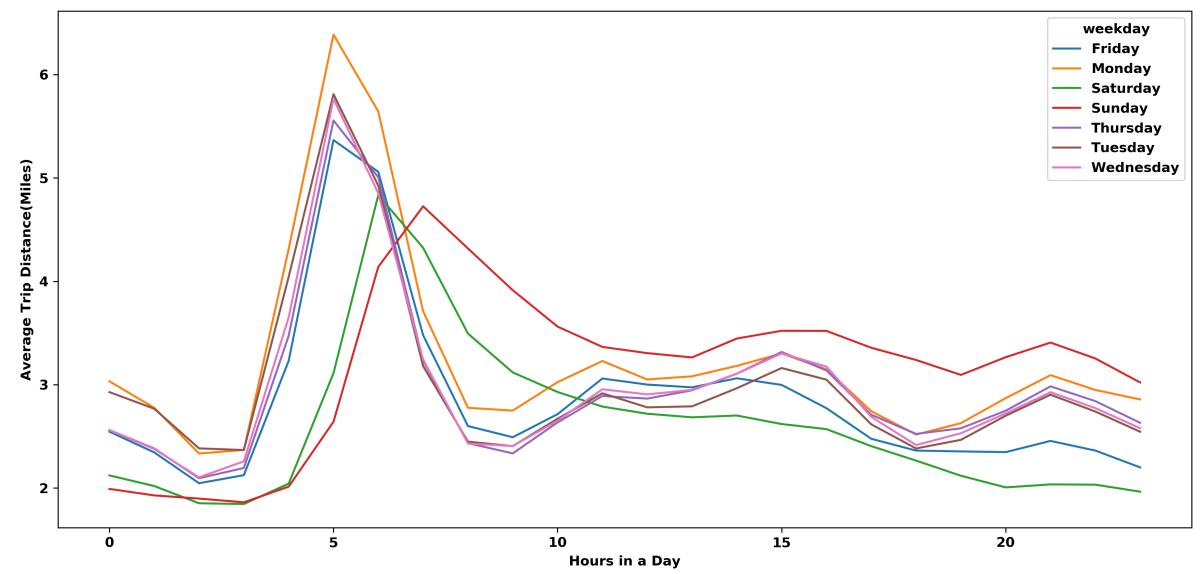

Figure 3.4: Average Trip Distance, by Hour of Day, for a Week

Monday morning.

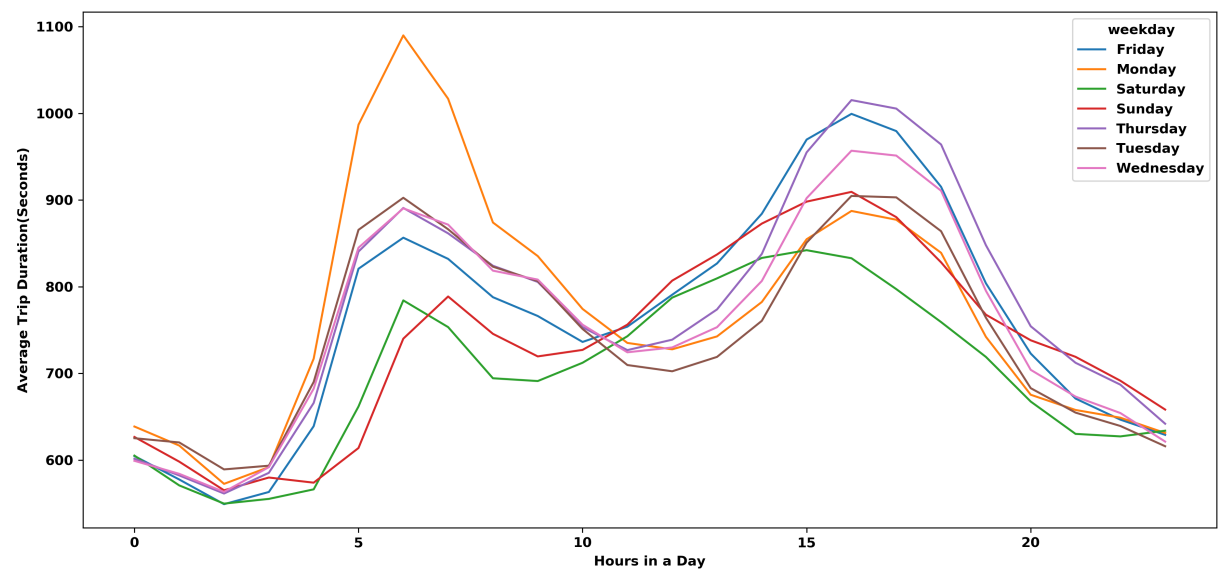

Figure 3.5: Average Trip Duration, by Hour of Day, for a Week

A data visualization was performed on the number of pick up requests recorded on special days. Fig 3.6 shows below shows an hourly count of pick up requests for three special days, i.e., Christmas Day, Independence Day and Valentines day. We see that the count is very high for Valentine's day (14 ${ }^{\text {th }}$ Feb 2016) which falls on Sunday for early hours of the day starting from midnight till early morning. The demand for taxi rises constantly at around 7-8 am till $7 \mathrm{pm}$ and then it drops slowly. The pattern is similar to Independences day which falls on Monday, however, during Valentines day, the number of requests during the peak hours is almost $1 / 3 \mathrm{rd}$ more than the Independence Day. The demands, however, rise after $9 \mathrm{pm}$ till midnight. This might be due to that fact that the next day is a working day, people are trying to head back home to that they can 
resume work from the next day. For Christmas day which falls on Sunday, the demand does not vary too much, there is no sharp increase or decrease. The demand is $1 / 6^{\text {th }}$ compared to Valentine's day, which is fairly low, although its a holiday. The low number of demands might be due to cold winter weather, or people generally prefer to stay indoors and celebrate holidays with families rather than heading out of their homes. For all the days the demand is low during early mornings, people do not want to venture out of their homes too early.

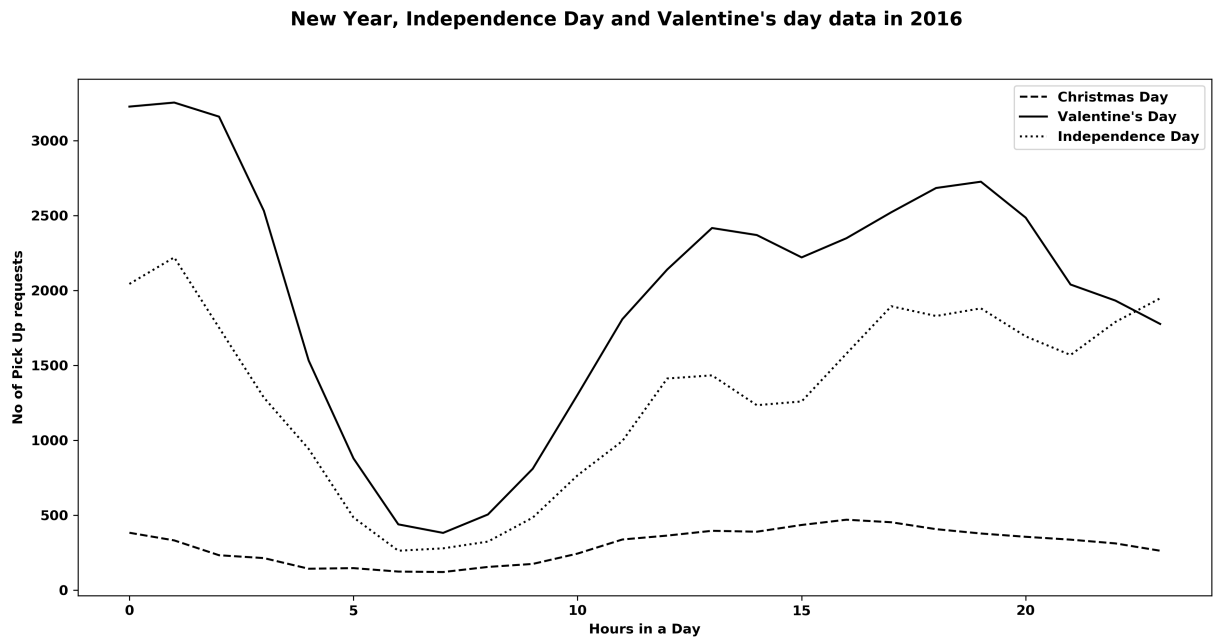

Figure 3.6: Total Trip Requests, by Hour of Day, for a Week

On assessing the differences based on the total number of requests average duration and average distance, a correlation between these days was observed. It can be summarized that the weekends have a high correlation for the trip patterns. 


\subsubsection{Temporal Analysis of the Pick-Up Requests}

We performed a temporal analysis for the pick-up requests from the taxi-set data to determine the peak and non-peak hours of requests for the rides. We can see the pattern through Figures 3.7, 3.8 and 3.9. On examining the distribution of the requests throughout the day, we were able to identify peak and non-peak hour patterns. This analysis was performed separately for 3 weeks of data in order to capture the trends accurately.

We have defined non-peak hours as numbers of ride requests which are less than 1000 within that hour or the lowest numbers within the 24 hour period. From the figures, we can identify non-peak hours between 2-7 am consistently on weekdays. However the patterns change over the weekends, and we notice non-peak hours between 6-8 am. The difference in hours suggests that on weekdays, people prefer to rest over the night hours as they have work during the day. While a shift on the non-peak hours during weekdays suggests that people most likely start their day late and prefer staying during the morning hours.

Similarly, we identified the peak hours during the weekdays typically fall between 8-9 am, 12-2 $\mathrm{pm}$ and 4-7 pm. The peak hours are the higher numbers of requests observed during the 24 hour of the day. These patterns suggest that people are trying to get to work in the mornings, or go for lunch and return home in the evenings. A spike in the number of requests is seen in the evening slot (4-7 pm) which is the highest throughout the day. We also notice a distinct trend during the weekends for peak hours as well. On Saturdays nights the peak hours were seen between 12-2 am and 10-12 pm. While on Sundays, we noticed peak hours between 12-2 am only as these are the higher numbers ( greater than 1000 request per hour) observed during that 24 hour period. These trends point to the fact that people are likely to go out till late at night on weekends compared to weekdays.

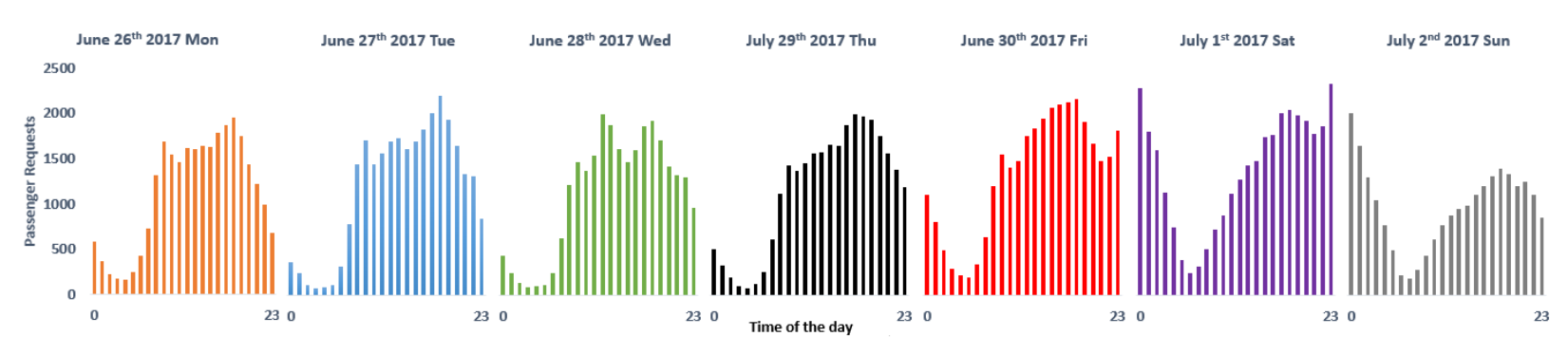

Figure 3.7: Passenger Requests Variations in Summer (June $26^{\text {th }}$ - July $2^{\text {nd }}$ 2017) 


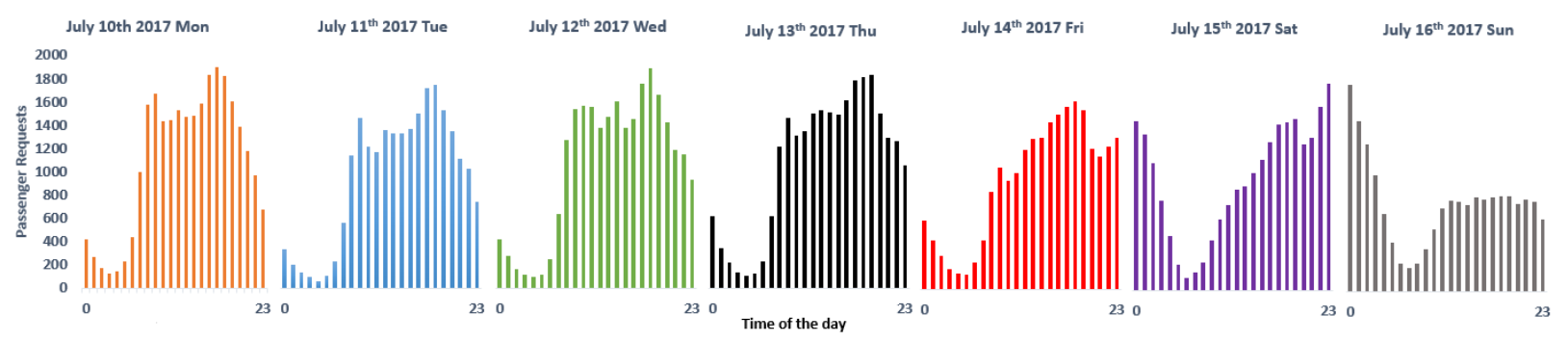

Figure 3.8: Passenger Requests Variations in Summer (July $10^{\text {th }}-16^{\text {th }} 2017$ )

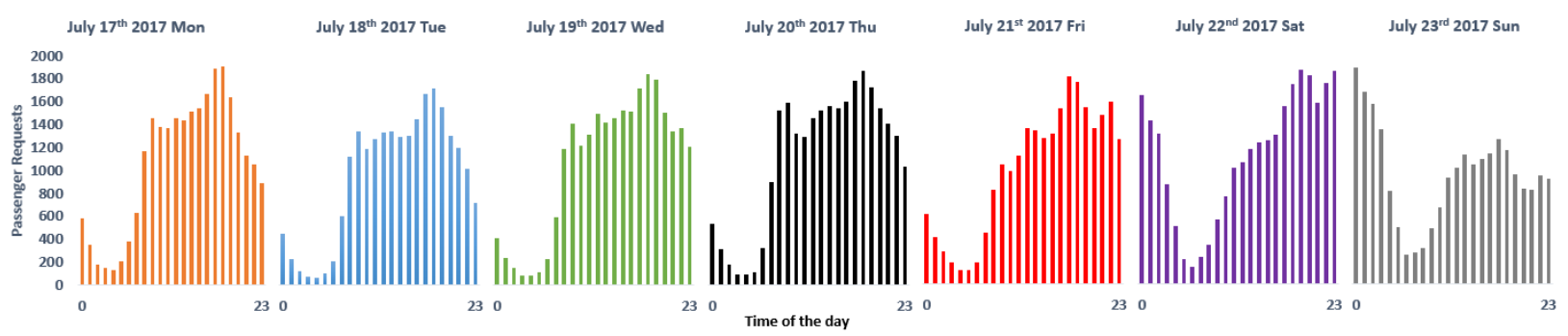

Figure 3.9: Passenger Requests Variations in Summer (July $17^{\text {th }}-23^{\text {rd }}$ 2017)

\subsubsection{Spatial Analysis of the Pick-Up Request in Chicago City}

We performed a spatial analysis of the Chicago dataset to understand the distribution of requests throughout the Chicago metropolitan area. We used the same set of data from the temporal analysis for a summer week and plotted the requests as shown in 3.10. The same data trend is observed during earlier years and later years as well. On close examination, we found the ride request distribution is highly uneven. A high concentration of ride is being originated and terminated into very few community areas. A very high number of rides are found in the Chicago downtown area, while the distribution is sparse on the other areas of the city. An outlier can be observed at Chicago O'Hare airport which also seems to experience a large number of requests. The high volume of requests is either seen in the airport, considering Chicago is a major city in the United States as well as downtown area that has a number of popular tourist destinations.

The temporal analysis helped us understand the different nature of ride requests fluctuating with each hour of the day. Therefore we designed our experiments for capturing the behavior of the system separately for peak and non-peak hours. It can be expected that the system is likely to behave uniquely for these two groups. An interesting insight provided by spatial analysis is the concentration of ride requests in certain areas. Thus we design our experiment to favor taxis already matched with passengers over taxis with no passengers. This 'rich getting richer' design is to help our system get more passengers in a single taxi. This improves the chances of a pooled or shared-ride which we are striving to achieve. 


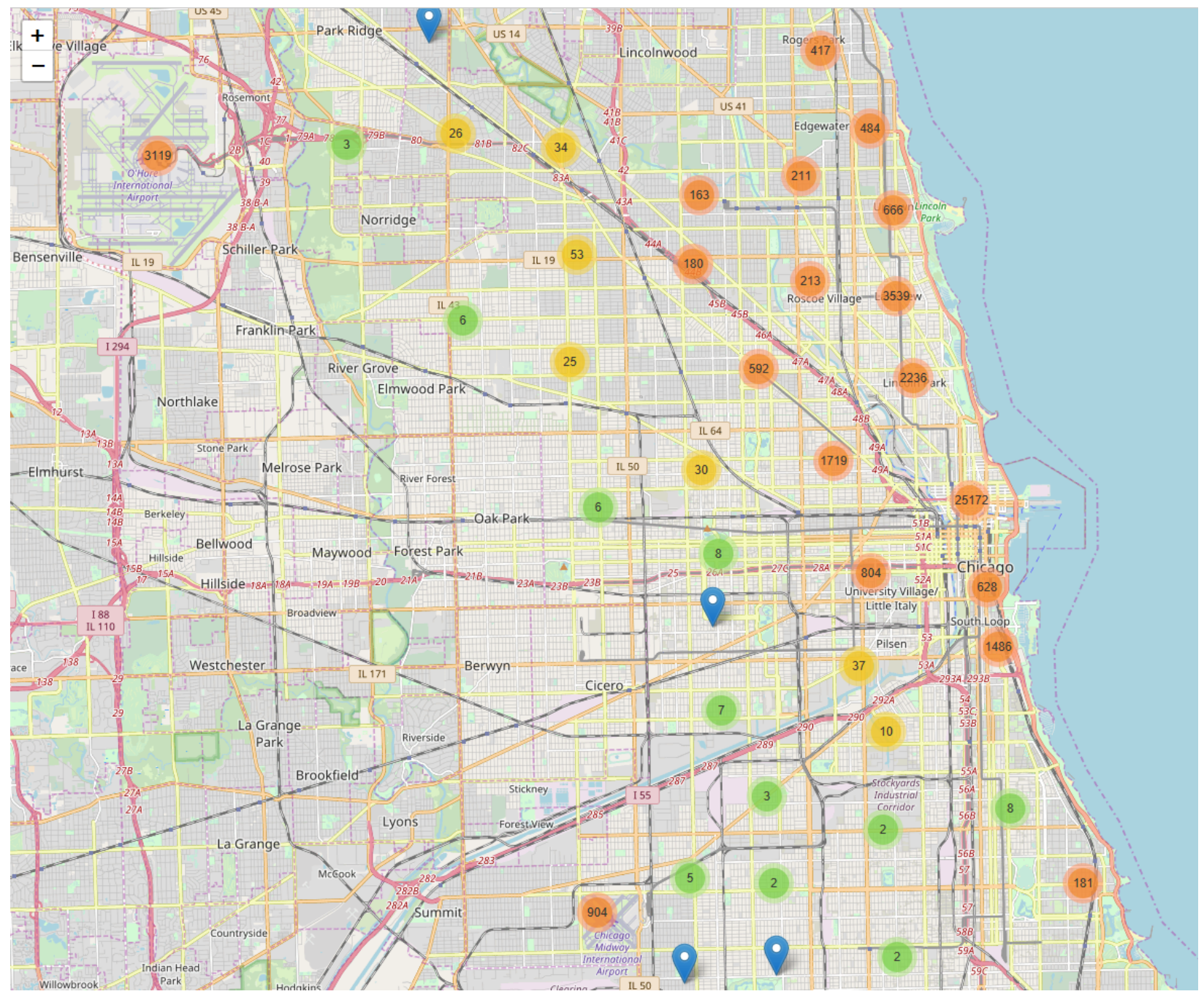

Figure 3.10: Pick Up Requests Distribution around Chicago city 


\section{Chapter 4 : Proposed Method: Dynamic Ride Sharing System using Particle Swarm Optimization}

Fig 4.1 shows a high level view of the proposed ride-sharing system. The communication between the entities are occurring in the form of request and response.

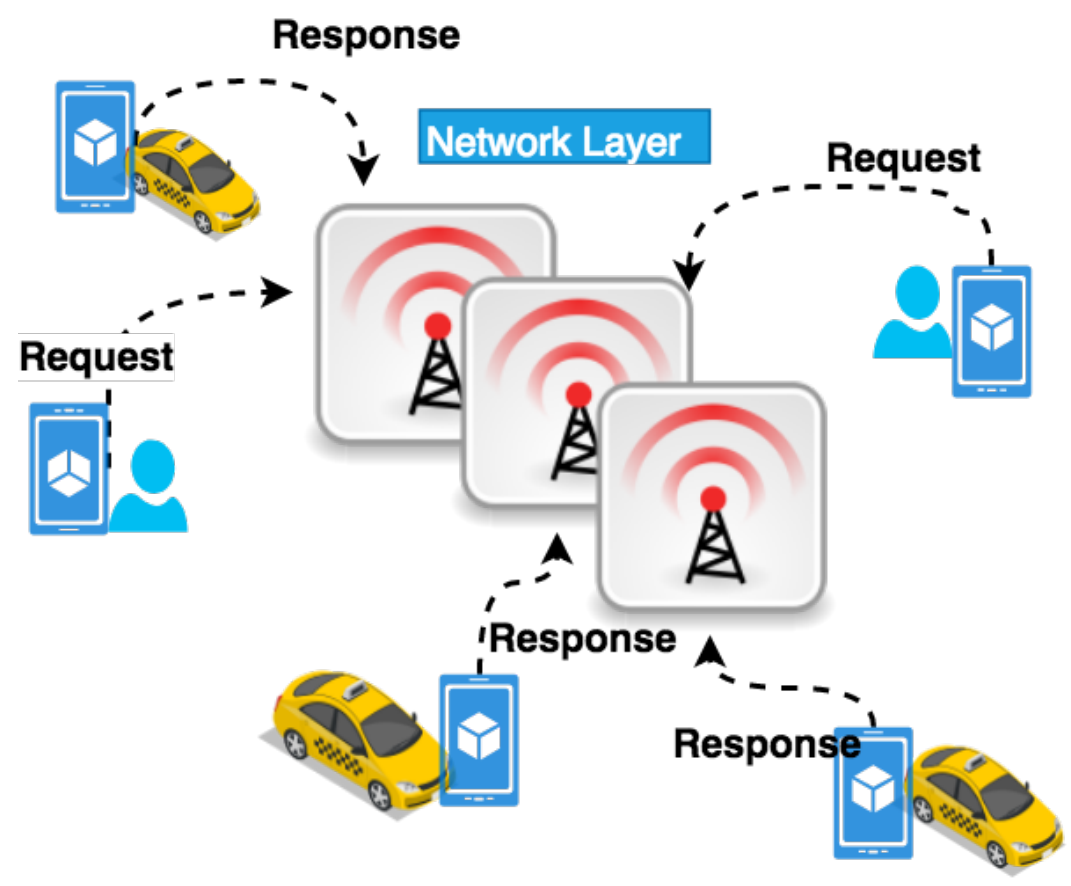

Figure 4.1: High Level View of a Ride Sharing System

The main entities involved in a ride-sharing system are:

- Passengers/Riders: The passengers are the human entity requesting the ride-sharing service for traveling from one place to another.

- Taxi: The taxi is the vehicle that offers to transport passengers from one location to another. In case of many passengers trying to travel together sharing the ride, the taxi can accommodate multiple requests based on its availability/capacity.

- Route: The path that a taxi follows while transporting the passengers.

- Event: There are two events, pick up and drop off events, associated with every request.

The variables definitions and their significance is discussed in the Table 4.2. We will be using these variables in the following sections .

A conceptual depiction of the simulation is provided in Fig 4.2. A hybrid model was chosen for the system design. The ride requests are channeled into a queue, which is routed to the taxis in 


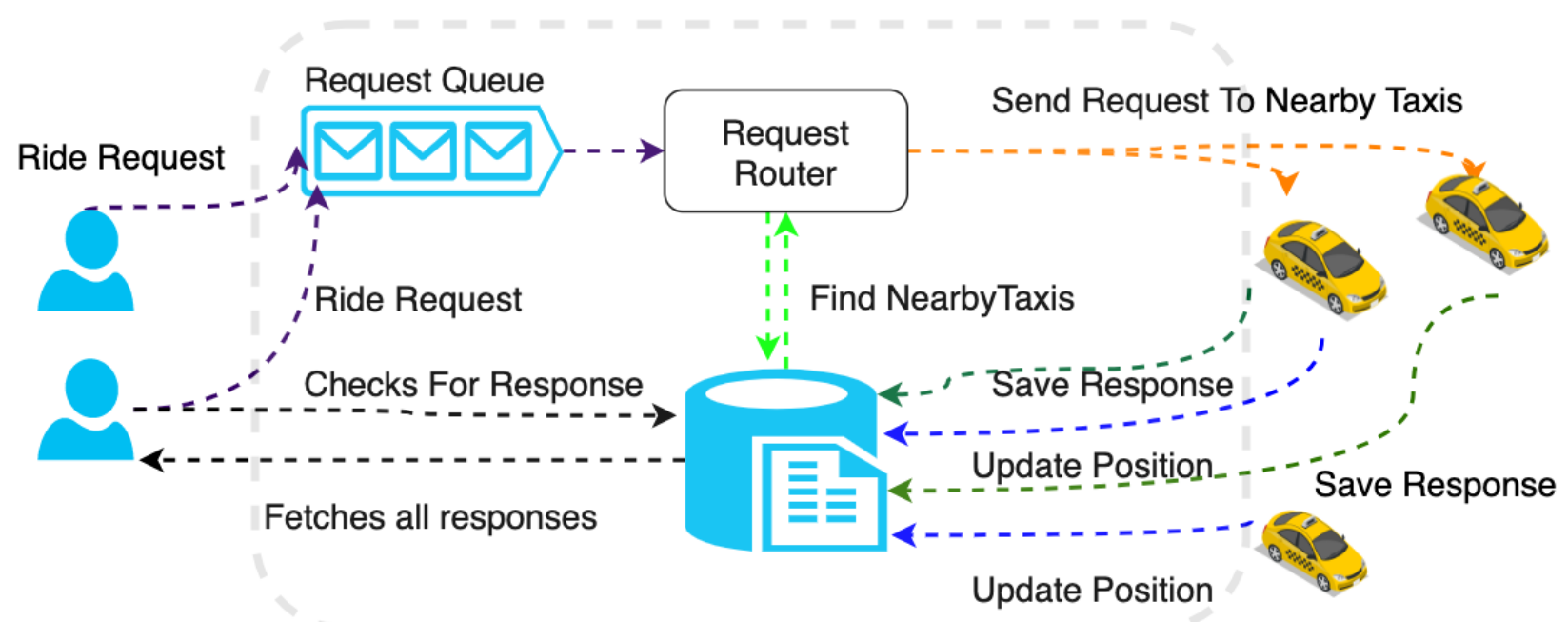

Figure 4.2: Simulation Platform

the vicinity (within a radius of $2 \mathrm{kms} / 3 \mathrm{kms}$ ). The platform models the behaviour of the passengers and vehicles and the communication between these components

We have made certain assumptions during the simulations, which are as follows:

1. The total number of passengers at any instant of time cannot exceed the taxi's capacity.

2. The pick up event always precedes the drop off event for every ride request in the taxi's schedule.

\subsection{Pseudo Code}

There are separate algorithms running at passenger's end and taxi's end. We will discuss the functions at each end in details in this section.

\subsubsection{Pseudo Code at Passenger's End}

At passenger's end, the REQUEST message is published to the messaging queue and is routed by the request router to all the taxis in the wireless range $(2 \mathrm{kms}$ or $3 \mathrm{kms})$. The waiting timer $w_{R_{p}}$ is started and enters a waiting state till the maximum wait time is exhausted. If the size of the REPLY queue is greater than zero, the best response is selected.The best response is selected based on the minimum cost, the number of passengers already assigned to the taxi (higher number preferred), the time and distance to reach from source to destination respectively. After the response is selected a CONFIRMATION message is sent to the taxi, to confirm the ride response. In case of no response, a new request has to be published. 
Table 4.1: Variable Definitions

\begin{tabular}{cc}
\hline Variables & Significance \\
\hline$P$ & Set of Passengers, passenger $p \in\{1,2,3, . . P\}$ \\
\hline$T$ & Set of Taxi, driver $t \in\{1,2,3, . ., T\}$ \\
\hline$S_{p}$ & Origin(source) location for $p$ \\
\hline$D_{p}$ & Destination for $p$ \\
\hline$t_{R_{p}}$ & Time at which p made a ride-sharing request \\
\hline$t_{R e_{p}}$ & Time at which p received a response \\
\hline$t_{\text {Pick }}$ & Time at which passenger p is picked up by taxi \\
\hline$t_{p o s}$ & Current position of taxi $t$ \\
\hline$t_{c a p}$ & Capacity of taxi $t$ \\
\hline$w_{R_{p}}$ & Waiting Time after sending request \\
\hline$M a x_{\text {wait }}$ & Maximum Wait Time \\
\hline$\Delta$ & Time to Travel from source to destination \\
\hline$t t_{s d}$ & 1, \\
\hline$x_{p}$ & if passenger $p$ is picked $u p$ \\
otherwise
\end{tabular}

Table 4.2: Messages Used in the Algorithm

\begin{tabular}{cc}
\hline Messages & Significance \\
\hline$R E Q U E S T\left(P_{i d}, S_{p}, D_{p}, t_{R_{p}}\right)$ & Request for ride-sharing sent by Passenger (P) \\
\hline$R E P L Y\left(T_{i d}, t_{\text {pick }}, t_{d r o p}, C_{p}\right)$ & $\begin{array}{c}\text { Reply from T to P with estimated pick-up time } \\
\text { drop off time and cost for the ride }\end{array}$ \\
\hline$C O N F I R M\left(P_{i d}, T_{i d}\right)$ & Acceptance/Rejection status sent by P to T \\
\hline$A C K\left(T_{i d}, P_{i d}\right.$, AckStatus $)$ & Acceptance/Rejection status sent by T to P \\
\hline
\end{tabular}




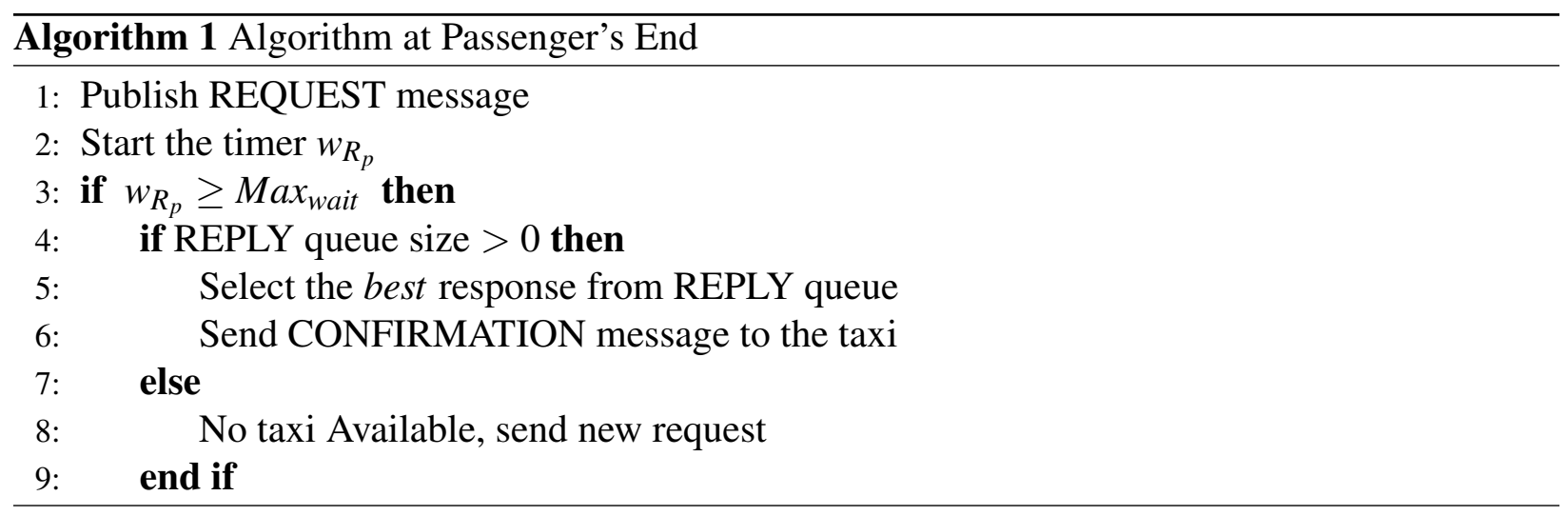

\subsubsection{Pseudo Code at Taxi's End}

At taxi's end, when a REQUEST message is received, the processing begins only if the taxi's maximum capacity is not reached. If the taxi can accommodate the incoming request, the feasibility of including the new request into the taxi's schedule is done by making a call to PSO for Route Optimization (Algorithm 3). If the taxi does not have enough seats to accommodate the incoming request, it does not call Algorithm 3. After the route is scheduled using Algorithm 3, the taxi sends a response REPLY message to the passenger. It starts the confirmation timer $T_{\text {confirm }}$ which expires in 90 seconds. In case a confirmation is received within 90 seconds of sending the response back to the passenger, the permanent schedule of the taxi and the taxi occupancy is updated. When the 90 seconds expires, the taxi is no longer waiting for confirmation but is now available to process new requests.

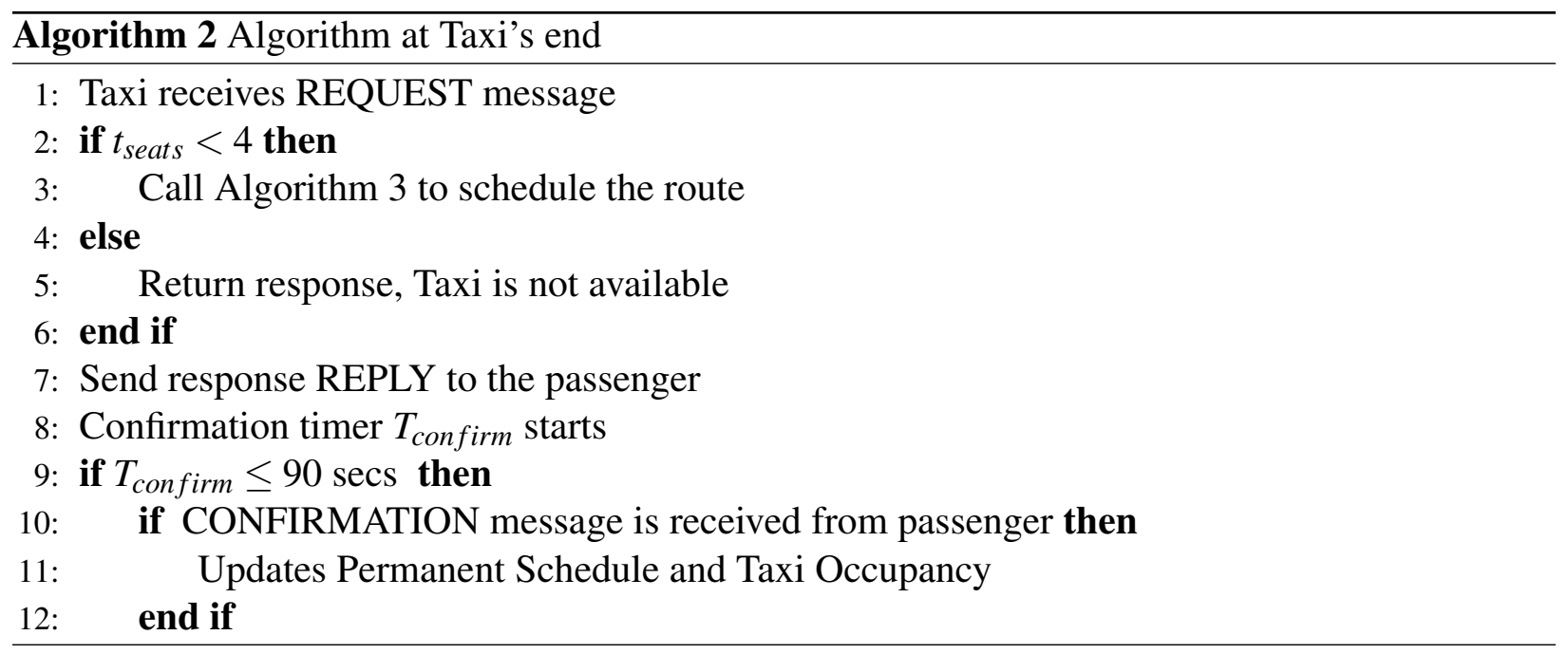

\subsubsection{PSO for Route Optimization}

Particle Swarm Optimization (PSO) is an evolutionary strategy inspired by the behavior of animals or birds. It helps in attaining the global minima of a given function. It works iteratively 
to converge a population of randomly initialized solutions also known as the particles, towards a globally optimal solution (global-best solution). Each particle within the population continues to track its current position and the best solution it has met, known as pbest. These particles have an associated velocity to span across the search space. The overall best solution of the swarm is called gbest, which is kept track of by the swarm itself. During each iteration, the swarm updates the velocity of the particle towards its pbest and gbest values.

Let $f(x)$ be the function to be minimized or in other terms the fitness function. In our case the fitness function is the Passenger Travel Time $\left(t t_{s d}\right)$. Passenger Travel Time is the total time taken for all the passenger to reach from source to destination including the detour caused by sharing the taxi with other passengers.

$$
\left.\min t t_{s d}=\sum_{p=1}^{P} x_{p} t \operatorname{ime}\left(S_{p}, D_{p}\right)\right)
$$

The objective functions are subject to all the constraints:

$$
\begin{gathered}
t_{\text {cap }} \leq 4, \forall t \in\{1,2,3, . ., T\} \\
w_{R_{p}} \leq \text { Max }_{\text {wait }}, \forall p \in\{1,2,3, . ., P\} \\
t t_{\text {sd }} \leq \operatorname{time}\left(S_{p}, D_{p}\right)+\Delta, \forall p \in\{1,2,3, . ., P\}
\end{gathered}
$$

The are three constraints $(4.2,4.3,4.4)$, defined above for the objective functions. $t_{c a p}$ is the condition for checking the taxi capacity, which should be less than 4. Equation 4.3 defines the waiting time $w_{R_{p}}$, which is the waiting time after the passengers requests for a ride and receives a response. This value should be smaller than the maximum wait time (Max wait $)$ which is predefined( for example 5, 10 minutes). Lastly, the time taken to travel $\left(t t_{s d}\right)$ from source to destination for any passenger, should not exceed the original travel time without sharing plus the detour tolerance $(\Delta)$.

The PSO algorithm updates the velocity and position of each particle during each iteration using the equations 4.5 and 4.6. Here, $x_{r}$ is the position of the particle and $v_{r}$ is the velocity of the particle for that position.

The particles are calculated using:

$$
\begin{gathered}
v_{r}=\omega v_{r}+c_{1} \text { rand }_{1}\left(\text { pbest }_{r}-x_{r}\right)+c_{2} \text { rand }_{2}\left(\text { gbest }_{r}-x_{r}\right) \\
x_{r}=x_{r}+v_{r}
\end{gathered}
$$

For each particle, two random numbers ( $\operatorname{rand}_{1}$ and $\mathrm{rand}_{2}$ ) are chosen from a uniform distribution that lies between 0 and 1. Omega $\omega$ is the inertia weight and $c_{1}, c_{2}$, which are the cognitive coefficients, which defines how much a particle can rely on its experience and the experience of its neighbors. In other words $c_{1}, c_{2}$ are the learning rates. In case a new position of the particle is a better fit than its pbest value, the algorithm updates the pbest to the new position. Once these iterations of pbest values are completed, the algorithm converges to a overall optimal or gbest value. The algorithm is finally terminated when the gbest has not changed by the end of an iteration, or the algorithm has attained the maximum number of iterations. The algorithm 3 describes the 
process in various steps.

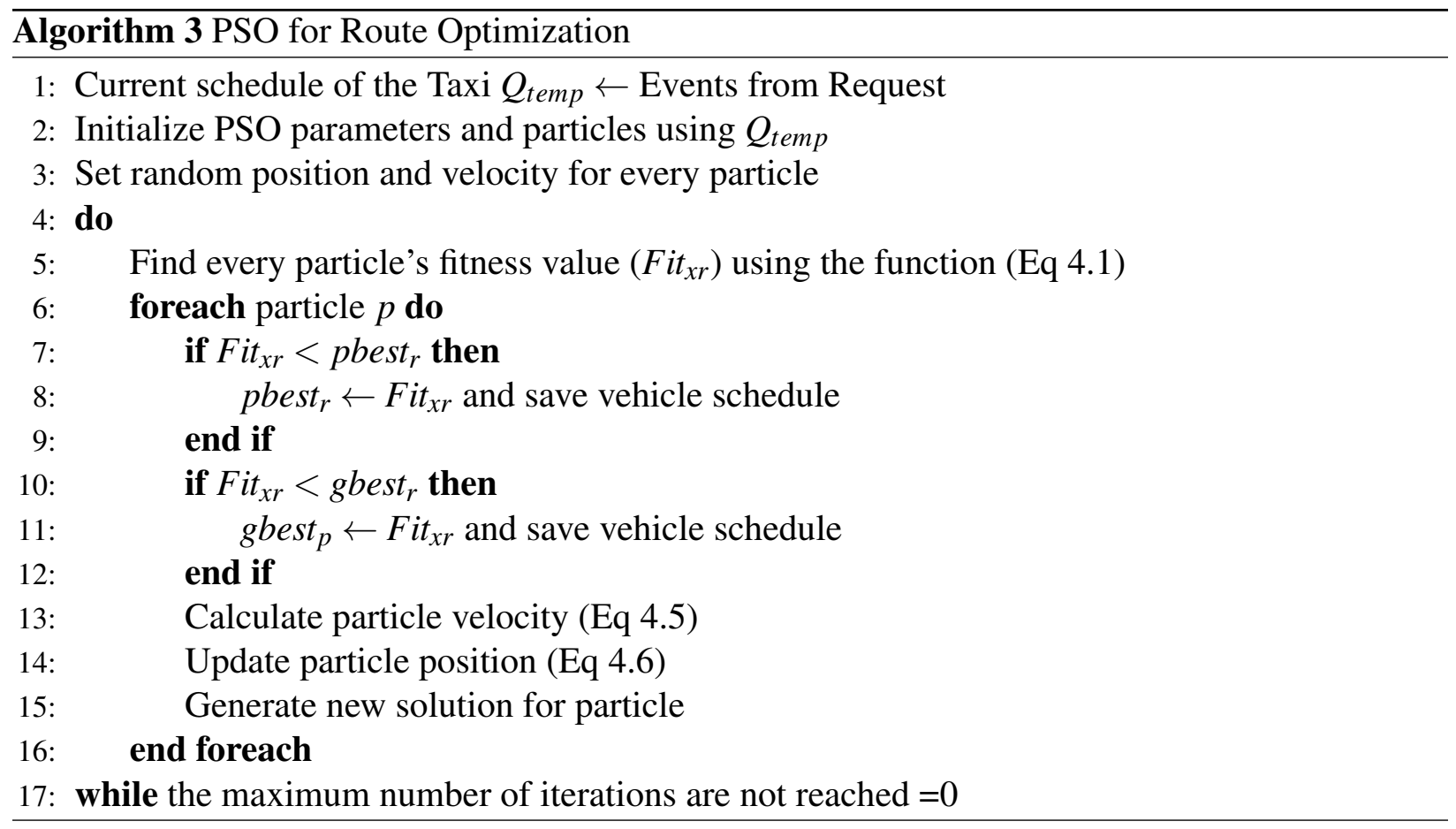




\section{Chapter 5 : Results and Validation}

In this chapter we describe our experimental design and setup, the results obtained, and the conclusions gathered from those results.

\subsection{Experimental Design}

The simulation parameters chosen for the experiments are listed in Table 5.1. We have set a constant capacity of 4 for all the taxis in the RSS. The taxi search radius for any incoming request is varied to $2 \mathrm{~km}$ (kilometers) and $3 \mathrm{~km}$ for performance comparison. The maximum delay (detour tolerance) a user can concede while sharing the ride is set to 10 minutes. The detour tolerance can be defined as the maximum delay a passenger is willing to handle for sharing the ride should not exceed 10 minutes. The particle count and the maximum number of epochs for the PSO algorithm are set to 100 each.

Table 5.1: The Simulation Parameters

\begin{tabular}{|l|c|}
\hline Parameters & Values \\
\hline Capacity of each Taxi & 4 \\
\hline Taxi search radius (passenger-centric) & $2 \mathrm{~km}, 3 \mathrm{~km}$ \\
\hline$\Delta$ (Detour Tolerance) & 10 minutes \\
\hline Particle Count & 100 \\
\hline Maximum Number of Epochs & 100 \\
\hline
\end{tabular}

We developed the simulation using Java and Python. We used two machines for the simulation. One of the machine is an Intel(R) Core(TM) i7-3770 CPU (3.40GHz) Linux machine (version 4.15.0-52-generic) with 12 GB RAM. The other machine is an Intel(R) Xeon(R) W-2133 CPU (3.60GHz) Windows machine with $128 \mathrm{~GB}$ RAM. Apache Kakfa ${ }^{1}$ is used for message passing and Redis ${ }^{2}$ is used for in-memory data storage. Routing APIs [46][47] were used to calculate the distance and time between the source and destinations. POSTMAN [48] is used for posting ride requests and registering taxis. The taxi clients, passenger clients, and router request applications were simulated as multiple processes running on different ports in these two machines.

We performed a simulation using real-world data from the Chicago Taxi dataset [12]. The CSV data file is approximately $43 \mathrm{~Gb}$, capturing the data from 2013 till August 2017. The dataset consists of 24 columns, and the total number of records is 112,860,054. This dataset contains single ride records. For each day of the week, non-peak hour data and peak hour data were extracted. The non-peak hour was repeatedly between 4-5 am time slot, while the peak hour was from 6-7 pm. However, we observed a different peak and non-peak hour during the weekends. During the nonpeak hour, a notable low number (100-250) of overall requests were created. Meanwhile, during

\footnotetext{
${ }^{1}$ Apache Kakfa: https://kafka.apache.org/

${ }^{2}$ Redis: https://redis.io/
} 
peak hour, a higher number (1900-4200) of overall requests were created. Those requests were satisfied by a particular number of individual taxis during that hour as observed from our dataset.

For our experiments, we used the original set of taxis that served those requests as well as injected additional taxis in various spots defined as taxi sets 1 through 4.The additional taxis These spots were determined by spatial analysis of the requests being generated during that hour. Through the afore-mentioned spatial analysis as seen in Section 3.2.2, spots of high demand and low demands were realized. Any spot with more than five requests originating at any point in time was marked as spots with higher demand. Whereas spots with less than five requests at any time were marked as spots of lower demand. Based on this principle, we prepared four sets of experimental taxi data with varying numbers:

- Taxi Set 1: The first set of taxi data were the taxis $\left(T_{o g}\right)$ that originally served the requests as obtained from the dataset. As mentioned earlier, these were single ride data, i.e., a single taxi was assigned to a single ride.

- Taxi Set 2: The second set consisted of Set 1 and additional taxis that were injected into the spots of low demands. A spatial analysis was performed for both peak and non-peak hour data for the ride requests as seen in section 3.2.2 to determine spots of low demands. A low demand spot was identified through the spatial analysis as a spot with less than or equal to 5 ride requests within that hour. For every such spot, an individual taxi was added.

- Taxi Set 3: The third set consisted of Set 1 with additional taxis that were added to the spots of high demands. A similar spatial analysis as in section 3.2.2 was performed to ascertain spots of high demands as well, for both peak and non-peak hours. Since we considered a spot with 5 and fewer requests as low demand spot, adversely a high demand spot is a spot with more than 5 ride requests within that hour. For every such spot, an additional taxi was simulated at that spot. This set had more taxis compared to Set 1 and Set 2.

- Taxi Set 4: The final set of taxi data was twice the taxis $\left(T_{o g}\right)$ that originally served the requests as obtained from the dataset i.e., $2 \times T_{o g}$. In other words, we simulated twice as much taxis for that hour (both peak and non-peak) irrespective of high or low demands. This meant a lot of taxis were available to support the incoming ride requests. This was the largest set of taxi data set used for performing the experiments.

The third set consists of the original set along with additional taxis injected in spots of higher demands. Finally, the fourth set contains twice the original number of taxis i.e., the first set. The spots of higher and lower demands were obtained by clustering the requests using spatial clustering. By simulating additional taxis in sets 2, 3 and 4, we wanted to study how the system behaves in such cases. The additional taxis can either help to improve the performance of the system and reduce it further. By carrying out experiments with these separate sets, we would be able to draw more definite results on our RSS's behavior. The experiments were performed using the four sets of taxis described above with a variation in their search radius of $2 \mathrm{~km}$ and $3 \mathrm{~km}$. We performed experiments for every day of a week and closely monitored the behavior of the ride-sharing system for these varying sets of simulated inputs. 


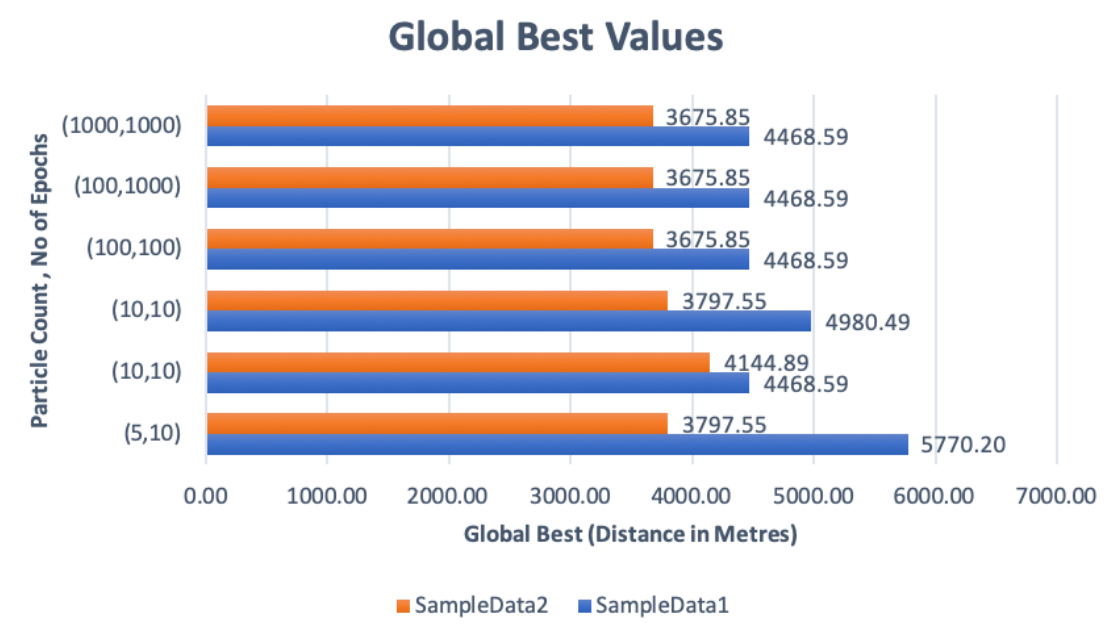

Figure 5.1: Global Best Values for a Combination of Particle Numbers and Epochs

To select appropriate values for particle count and the maximum number of epochs for the PSO algorithm implementation as shown in the Table 5.1, several test runs were performed using two separate sets of sample data. The values of the particle used were 5, 10, 100 and 1000. Similarly, a set of values such as 10, 100 and 1000 were chosen for the maximum number of epochs. While making the decision the results of gbest (global best) position were observed, as seen in Fig 5.1. From the results obtained, it was observed that the gbest values do not improve after the 100, 100 combination but remain rather constant. Although the remaining combinations such as 100, 1000 and 1000, 1000 also provided the same gbest value it took more execution time compared to the 100, 100 combinations. This 100 was chosen as ideal value for performing experiments for both particle count and epochs in the PSO algorithm for routing optimization. Various combination of these values was used and finally, 100 was chosen for both the particle count and the number of epochs.

\subsection{Performance Metrics}

The results were calculated based on the metrics designed for the proposed system for each run (non-peak hour and peak hour).

\section{Overall Success Rate of Ride Matching (OSR)}

The ratio of the total number of rides matched to the taxis by the system to the total number of ride requests made by the passengers. OSR also includes the taxis that are matched to pick only a single passenger.

$$
O S R=\frac{\sum_{p=1}^{P} M R_{p}}{\sum_{p=1}^{P} R_{p}} \times 100
$$

\section{Success Rate of $N^{\text {th }}$ Passenger (SRNP)}


Defined as the success rate of matching first, second, third and fourth $(N=\{1,2,3,4\})$ passengers to the taxis. This helps to visualize the distribution of requests among available taxis in the ride-sharing system. The SRNP value for more than one passenger $(\mathrm{N}>1$ and $\mathrm{N}$ $\leq 4)$ helps to define the effectiveness of the proposed ride-sharing system.

$$
\begin{aligned}
& \operatorname{SRNP}_{\{N=1\}}=\frac{\sum_{p=1}^{P} M R_{p 1}}{\sum_{p=1}^{P} R_{p}} \times 100 \\
& \operatorname{SRNP}_{\{N=2\}}=\frac{\sum_{p=1}^{P} M R_{p 2}}{\sum_{p=1}^{P} R_{p}} \times 100 \\
& \operatorname{SRNP}_{\{N=3\}}=\frac{\sum_{p=1}^{P} M R_{p 3}}{\sum_{p=1}^{P} R_{p}} \times 100 \\
& \operatorname{SRNP}_{\{N=4\}}=\frac{\sum_{p=1}^{P} M R_{p 4}}{\sum_{p=1}^{P} R_{p}} \times 100
\end{aligned}
$$

\section{Distance Ratio (DR)}

The ratio of the sum of individual trip distances between pick-up and drop-off locations for all passengers to the total distance traveled with more than one passenger.

$$
D R=\frac{\sum_{p=1} \operatorname{dist}\left(S_{p}, D_{p}\right)}{\sum_{p=2,3,4} \operatorname{dist}\left(S_{p}, D_{p}\right)} \times 100
$$

\section{Average Passenger Waiting Time (AWT)}

The average difference in time between the instant a passenger request is submitted and the instant a taxi picks up the passenger.

$$
A W T=\frac{t_{P i c k}-t_{R_{p}}}{\sum_{p=1}^{P} R_{p}}
$$

\section{Average number of messages (ANM)}

It is defined as the ratio of the total number of messages that are sent and received in the ride-sharing system to the total number of confirmed (successful) ride requests for an hour.

$$
A N M=\frac{\sum_{p=1}^{P} T_{m e s s a g e s}}{\sum_{p=1}^{P} R_{p}}
$$

\section{Average Request Processing Time (ARPT)}

The average difference in time between the instant a passenger ride request is submitted and the instant a response from the ride-sharing system is received. This wait time is less than 
the maximum wait $\left(\right.$ Max $\left._{\text {wait }}\right)$ time.

$$
A R P T=\frac{t_{R e_{p}}-t_{R_{p}}}{\sum_{p=1}^{P} R_{p}}
$$

\subsection{Results for Summer Week and Results for Winter Week}

Experiments were conducted separately for the non-peak hours and peak hours for every day of a week in summer ( July $10^{\text {th }}$ - July $16^{\text {th }} 2017$ ) and a week in winter ( Dec $12^{\text {th }}-$ Dec $16^{\text {th }}$ ) as shown in the following sections.

\subsubsection{Results for Summer Week}

We recorded the results for both peak and non-peak hours in three groups, i.e., weekdays ( Monday- Thursday), weekends (Friday and Saturday) and Sunday. The overall success rate (OSR) for the non-peak hour is shown in Fig 5.2. Fig 5.2a is the success rate for weekdays, the maximum value is $97.05 \%$ which is obtained for taxi set 2 for a taxi radius of $3 \mathrm{~km}$. Taxi set 1 (i.e., the actual number of taxis) also performs efficiently with 92.53\%. As the number of taxis increased gradually from taxi set 1 to taxi set 4 , we observe that the OSR increases. The OSR also increases with a wider taxi search range of $3 \mathrm{~km}$, as the ride request can reach a larger number of taxis. Similarly, the ride-sharing pattern for the weekend is captured in Fig 5.2b. We can see that the OSR is significantly improved for taxi set 3 which is observed at $99.45 \%$. The results are improved by injecting additional taxis in the spots of more demands. The OSR rate during Sunday differs from the weekends (Fri-Sat) patterns, as seen in Fig 5.2c. The injection of additional taxis in taxi sets 2,3 and 4 does not improve the OSR.

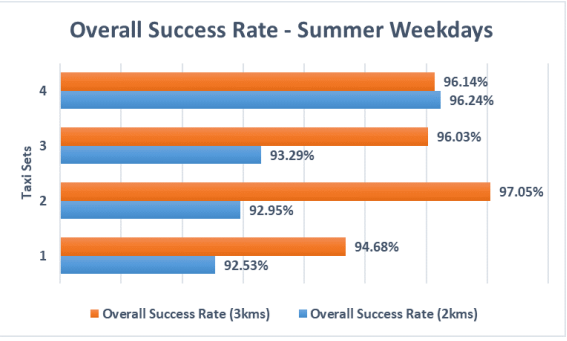

(a)

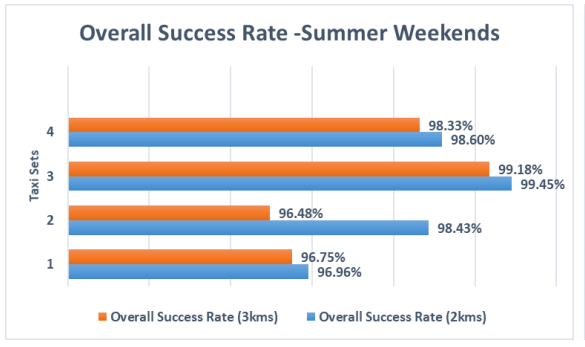

(b)

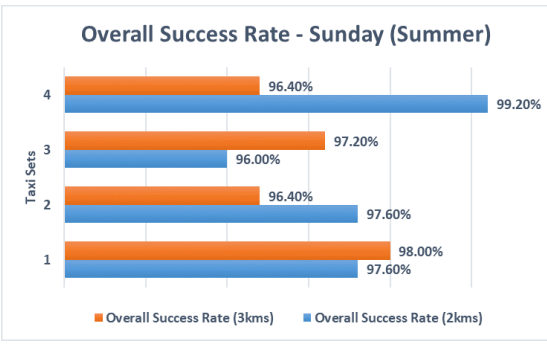

(c)

Figure 5.2: Overall Success Rate (OSR) of Ride Sharing Service (RSS) for Non-Peak Hours

The results for the peak hour is shown in Fig 5.3. It can be observed that the performance of the RSS during peak hours is not as good as that during non-peak hours. This is due to the an extremely large number of incoming requests. From Fig 5.3a we observe the OSR to be its maximum with $65.05 \%$ for taxi set 3. Similarly, for weekends and Sunday, the overall success rate during peak hour improves on injecting more taxis in the spots of more demands. However, the OSR suffers by increasing the taxi search radius from $2 \mathrm{~km}$ to $3 \mathrm{~km}$, as observed in the figure throughout. A 


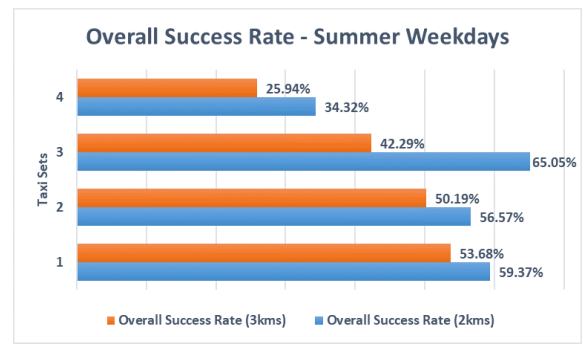

(a)

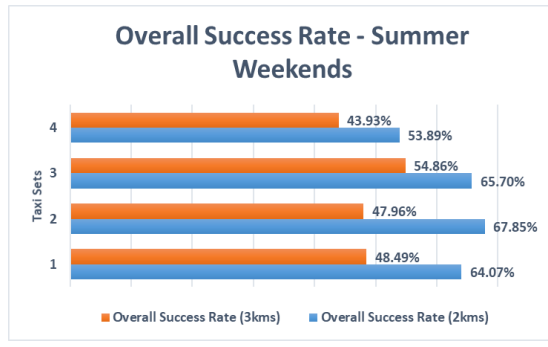

(b)

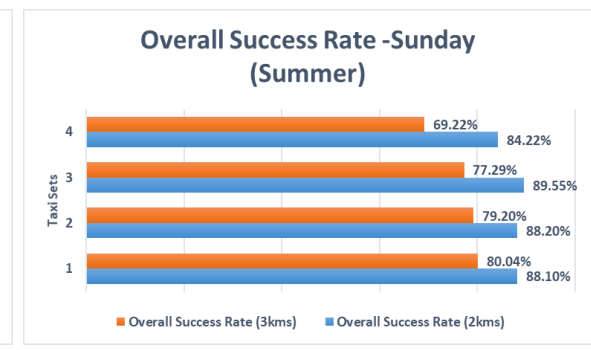

(c)

Figure 5.3: Overall Success Rate (OSR) of Ride Sharing Service (RSS) for Peak Hours

larger taxi radius sends the ride-sharing request to more number of taxis and requires additional computations for ride matching, thus deteriorating the performance of the system.
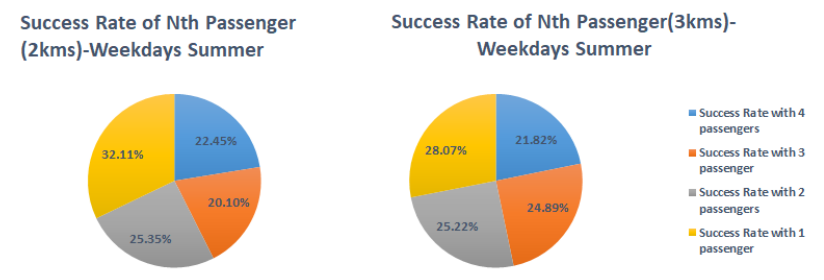

(a) Non-Peak Hours

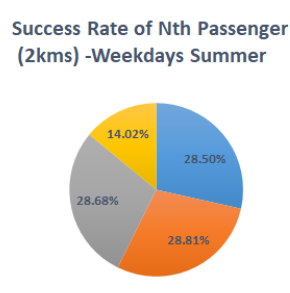

(b) Peak Hours

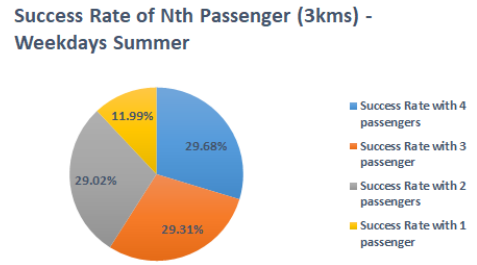

Figure 5.4: Success Rate of $N^{\text {th }}$ passengers (SNRP) on Weekdays

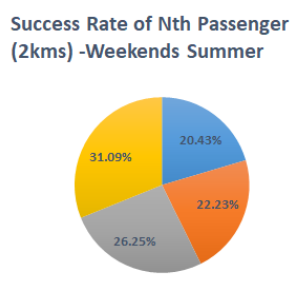

(a) Non-Peak Hours

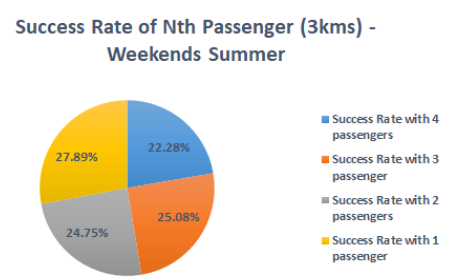

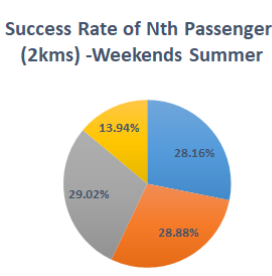

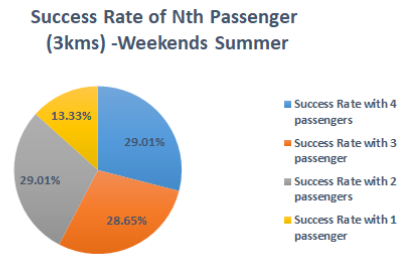

(b) Peak Hours

Figure 5.5: Success Rate of $N^{t h}$ passengers (SNRP) on Weekends

Fig 5.4, 5.5 and 5.6 show the success rate of $N^{\text {th }}$ passenger (SRNP). Through these figures we can understand how the confirmed rides are allotted to the taxis. We can see if our RSS is matching only single passengers to a taxi or is matching the new passenger requests to taxis with existing passengers en route to their destinations. The figures of the left hand side (5.4a, 5.5a and 5.6a) are for non-peak hours and we can see that the highest percentage is with 1 passenger. That means more ride requests are matched to taxi for non-shared rides. This pattern is similar for both $2 \mathrm{~km}$ and $3 \mathrm{~km}$ radius. This arises due to sporadic requests during non-peak hours, the RSS tries to match at least one passenger. We see that there is not a very large gap, the remaining rides are almost equally (above 20\%) shared by taxis having 2, 3 and 4 passengers as well. In contrast the 


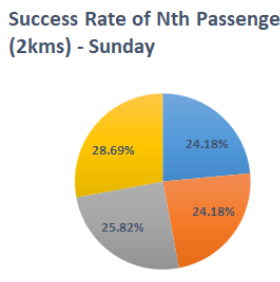

(a) Non-Peak Hours

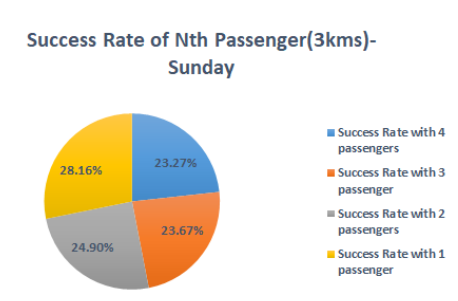


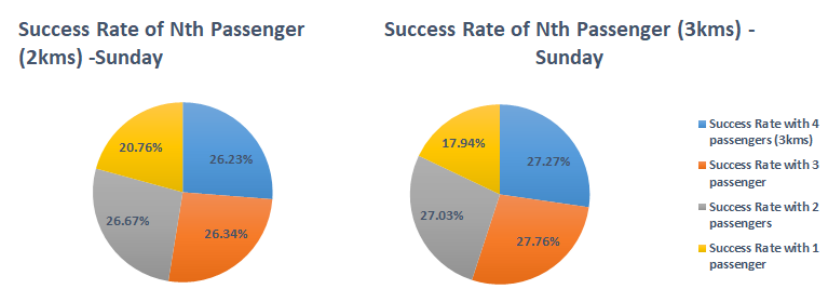

(b) Peak Hours

Figure 5.6: Success Rate of $N^{\text {th }}$ passengers on Sunday

behaviour of our RSS varies for the peak hours, we can see from the pie chart on the right hand side (5.4b,5.5b and 5.6b), less rides are allotted to taxis with only one passenger. The ride match for 1 passenger only in a taxi is as low as $11.99 \%$. We can also see for the other case i.e., with 2 , 3,4 passengers , the OSR is almost similar. This shows that during the peak hours the taxis already occupied by passengers are likely to accept incoming requests. This is because of the fact that, during peak hours more taxis as well are passengers are likely to be present in common locations and are easily matched.

The results for distance Ratio (DR) is captured in Fig 5.7 for the non-peak hours. We notice a consistent behaviour for all the three categories as well as the different combination of taxi sets with $2 \mathrm{~km}$ and $3 \mathrm{~km}$ radius. The DR value correlates to the extra travelling distance for shared rides compared to single rides. Thus a higher value for the distance ratio shows that there is a significant improvement on the total distance travelled with multiple passengers sharing their ride versus single rides. For both weekdays $5.7 \mathrm{a}$ and weekends $5.7 \mathrm{~b}$, the DR value is consistently better for taxi search radius within $2 \mathrm{~km}$. We can infer that if the taxis are nearer to the ride requests, there will be smaller deviation from their original path. Thus we obtain a better result for $2 \mathrm{~km}$ taxi search radius. For Sundays, as seen in 5.7c, the behaviour is consistent for taxi sets 1, 2 and 3 , however for taxi set 4 , the DR for $3 \mathrm{~km}$ performs better. The reason can be due to better positioning of taxis and similar routes for the data set on the particular date. The results for the peak hours are consistent with our observations for non-peak hours as seen in Fig 5.8a and Fig 5.8b. However, the pattern for Sunday is not consistent with our previous inferences as seen in Fig 5.8c. This is inferred to be caused by a different travelling habits of people during Sundays as compared to other weekdays or weekends. On Sundays people generally tend to stay in their homes in the earlier half, and may go out to church or public places with their families.

The average passenger wait time (AWT) for both non-peak and peak hours are captured in Fig 5.9 and Fig 5.10, respectively. We observe a small improvement in the AWT in case of taxi set 4 compared to the other taxi sets. This means that AWT improves when served by taxi sets that is twice the original set of taxi. However, this is not the case with peak hours, the AWT value drops (smaller value preferred) for taxi sets 2, 3 which means the average waiting time is improved when taxis are injected to spots of low and high demands. This shows that additional taxis improves the chances of serving the incoming requests faster. However, we also notice a sharp increase in AWT when the taxi search radius is increased to $3 \mathrm{~km}$. This means that the taxis at a further distance can serve the ride requests but will require additional time to reach the passenger to pick them up as 


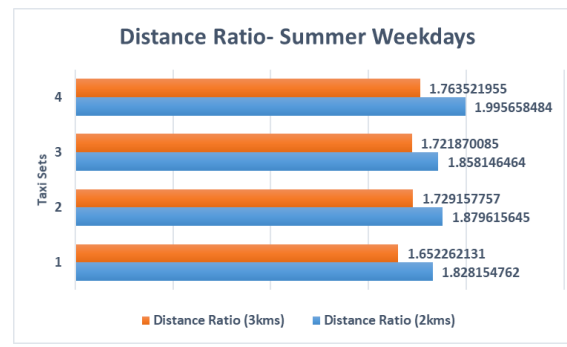

(a)

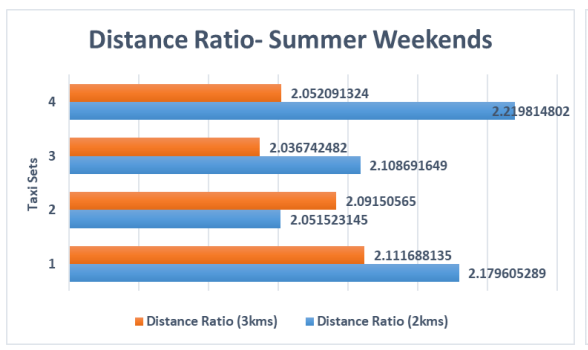

(b)

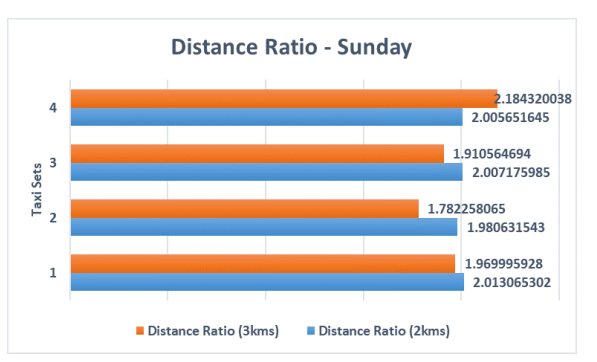

(c)

Figure 5.7: Distance Ratio (DR) for Non-Peak Hours

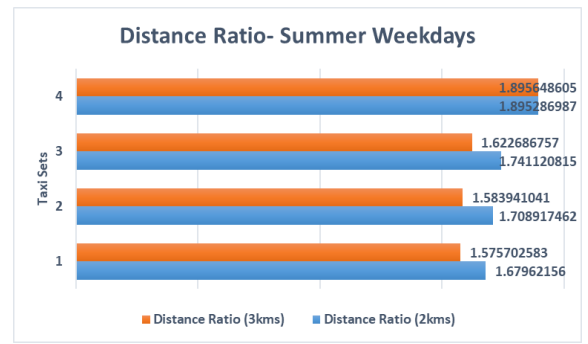

(a)

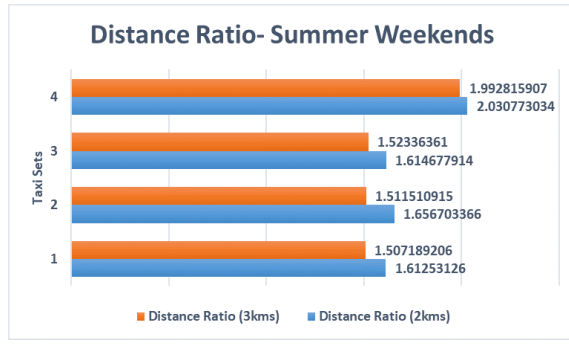

(b)

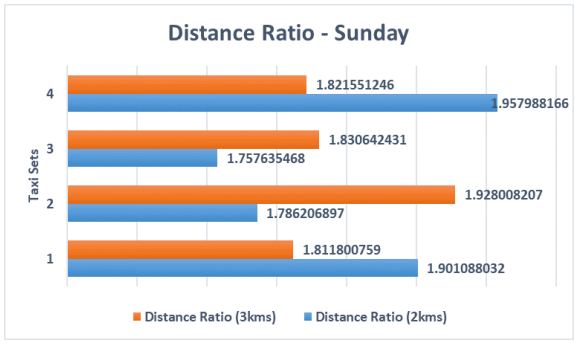

(c)

Figure 5.8: Distance Ratio (DR) for Peak Hours

compared to the taxis within $2 \mathrm{~km}$ radius.

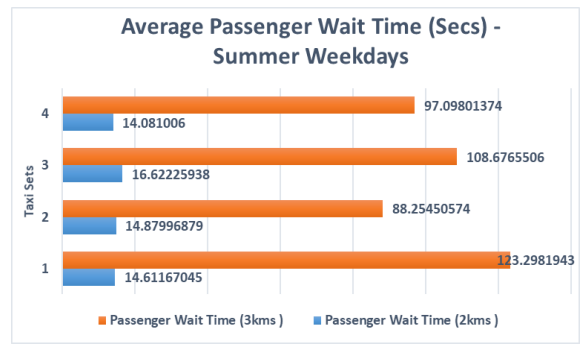

(a)

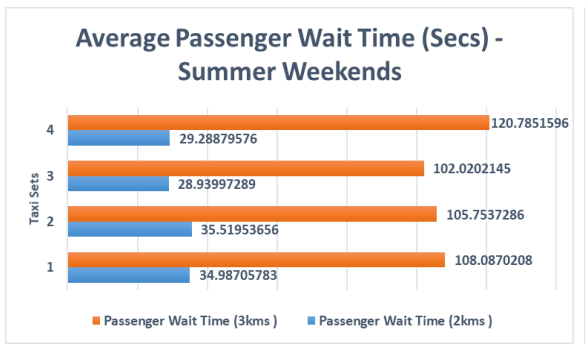

(b)

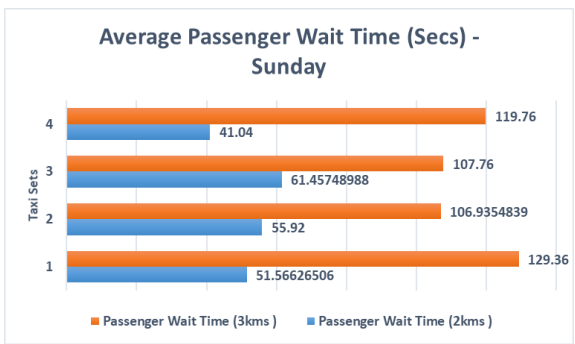

(c)

Figure 5.9: Average Passenger Wait Time (AWT) in Seconds for Non-Peak Hours

The average number of messages (ANM) exchanged in the RSS is as low as 6 to a maximum of 43 for non-peak hours as shown in Figures 5.11a 5.11b and 5.11c. With an increase in the number of taxis from taxi set 1 to 4, we notice the ANM also increases. Further the ANM for $3 \mathrm{~km}$ is more than for $2 \mathrm{~km}$ radius throughout the results obtained. This is because more taxis are available at a larger radius and hence more messages are sent back and forth to confirm the rides. From Fig 5.12, it is seen that ANM has much larger values as compared to non-peak hours, but even in this case the ANM value increases with more number of taxis as seen in taxi set 2, 3 and 4 . The minimum value for peak hour is observed at 238 for taxi set 1 during the weekdays and the maximum value is 671 for taxi set 4 with a $3 \mathrm{~km}$ taxi radius. The huge number of ANM is due to the large number 


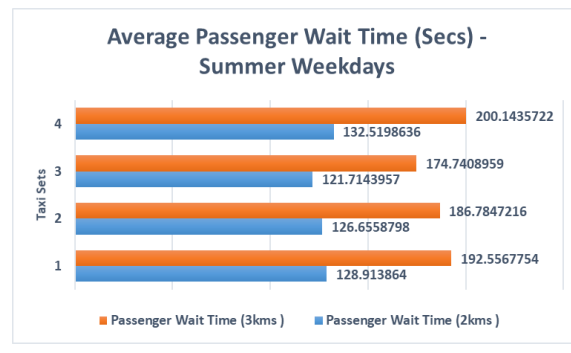

(a)

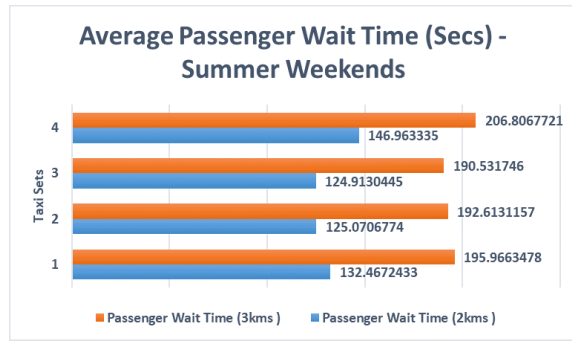

(b)

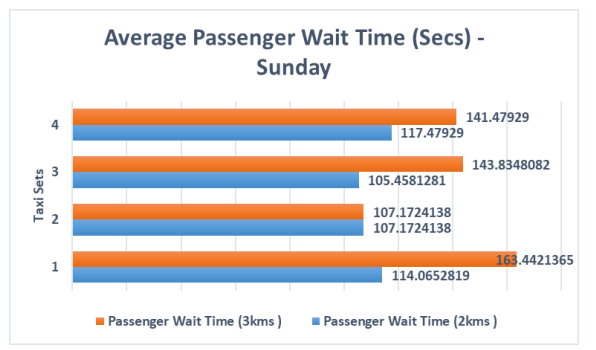

(c)

Figure 5.10: Average Passenger Wait Time (AWT) in Seconds for Peak Hours

of ride requests and equally larger number of taxis available to receive the ride requests.

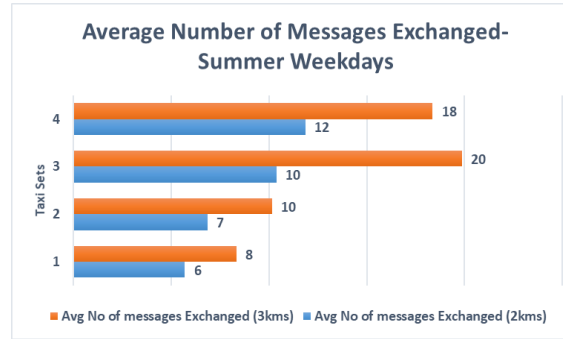

(a)

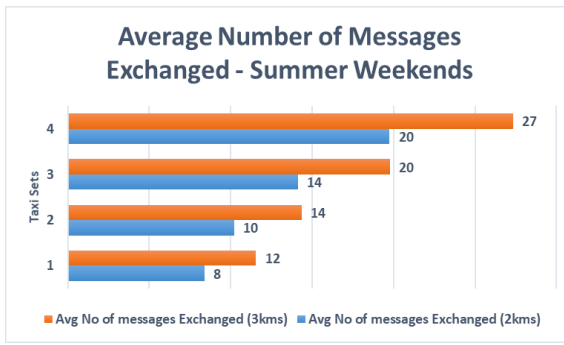

(b)

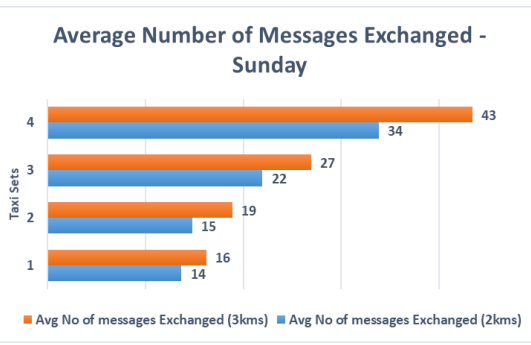

(c)

Figure 5.11: Average Number of Messages Exchanged (ANM)for Non-Peak Hours

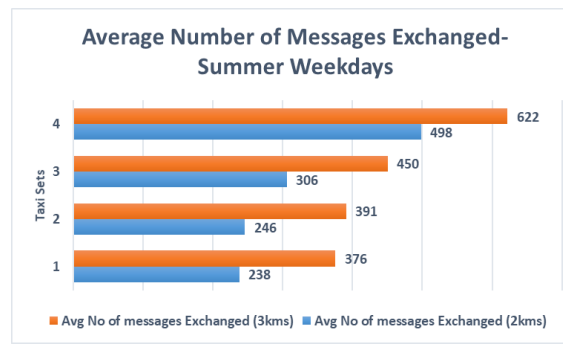

(a)

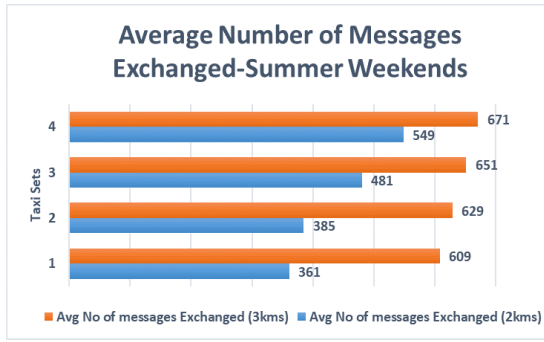

(b)

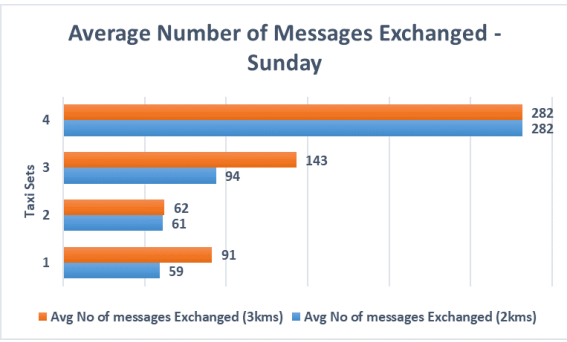

(c)

Figure 5.12: Average Number of Messages Exchanged (ANM) for Peak Hours

The average request processing time (ARPT) is for non-peak hours is shown in Fig 5.13 and for peak hours in Fig 5.14. It can be noticed from the figures that there are huge differences in ARPT values between different hours. Smaller ARPT values are observed for non peak hours with a minimum of $17.57 \mathrm{~ms}$. The increase in value occurs both due to additional taxis as well as increase in the taxi search radius. Similarly, an almost identical trend is observed for peak hours in terms of the growth in values for various taxi sets. However, the huge values of ARPT which ranges between $320-845 \mathrm{~ms}$ is understood to be due to the large number of requests which utilizes more resources for the complex computations and hence takes more time to process the requests. 


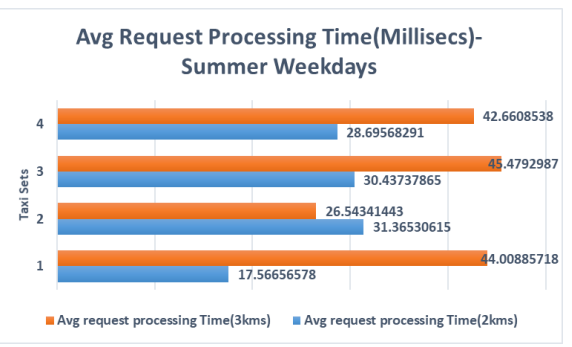

(a)

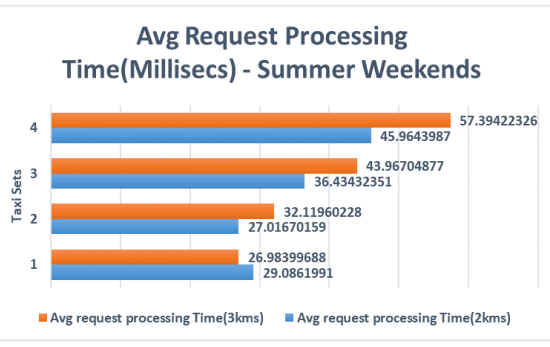

(b)

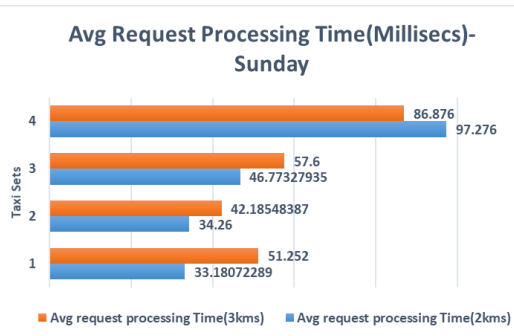

(c)

Figure 5.13: Average Request Processing Time (ARPT) in Milliseconds for Non-Peak Hours

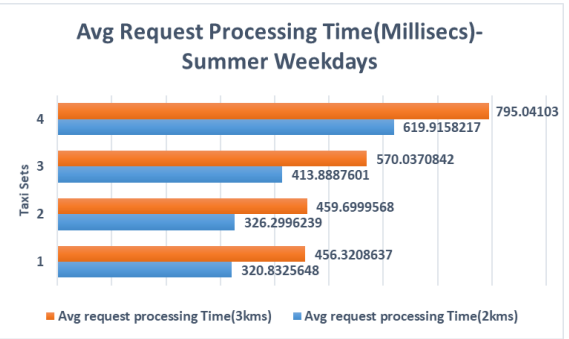

(a)

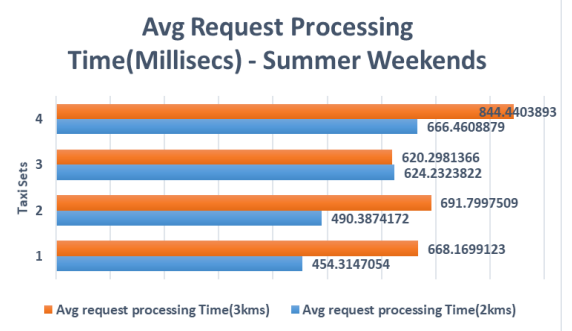

(b)

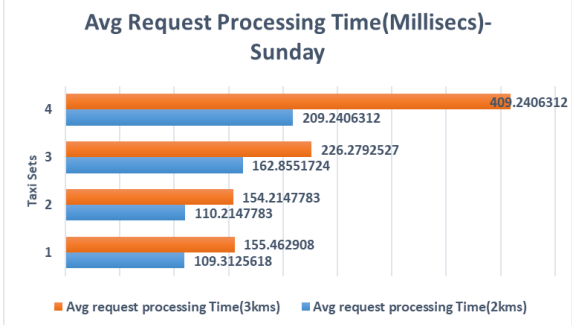

(c)

Figure 5.14: Average Request Processing Time (ARPT) in Milliseconds for Peak Hours

Thus, resulting into much higher values of ARPT. However, we can observe here that the actual request processing time is minimal (in milliseconds), which shows that our application provides very quick response for the experimental setup chosen in this case.

\subsubsection{Results for Winter Week}

We have conducted experiments for the winter week of Dec $12^{\text {th }}$ to Dec $16^{\text {th }}$. The overall success rate (OSR) for the non-peak hour is shown in Fig 5.15. The first graph,Fig 5.15a is the success rate for weekdays, the maximum value is $97.05 \%$ which is obtained for taxi set 2 for a taxi radius of $3 \mathrm{~km}$. Taxi set 1 (i.e., the actual number of taxis) also performs efficiently with $91.38 \%$. As the number of taxis increased gradually from taxi set 1 to taxi set 4 , we observe that the OSR increases. The OSR also increases with a wider taxi search range of $3 \mathrm{~km}$, as the ride request can reach a larger number of taxis. Similarly, the ride-sharing pattern for the weekend is captured in Fig 5.15b. We can see that the OSR is significantly improved for taxi set 3 which is observed at $99.45 \%$. The results are improved by injecting additional taxis in the spots of more demands. The OSR rate during Sunday differs from the weekends (Fri-Sat) patterns, as seen in Fig 5.15c. The injection of additional taxis in taxi sets 2, 3 and 4 does not improve the OSR.

The results for the peak hour is shown in Fig 5.16. It can be observed that the performance of the RSS during peak hour is not as good as that during non-peak hour. This is due to the an extremely large number of incoming requests. From Fig 5.16a we observe the OSR to be its maximum with $66.34 \%$ for taxi set 3 . Similarly, for weekends and Sunday, the overall success rate during peak hour improves on injecting more taxis in the spots of more demands. However, 


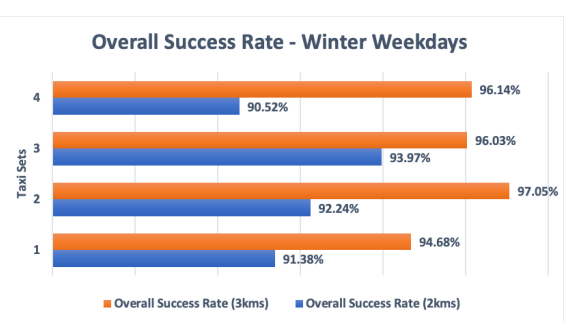

(a)

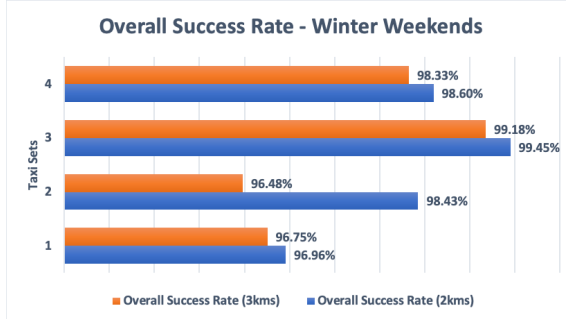

(b)

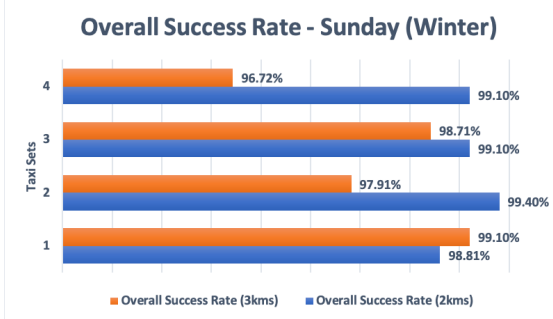

(c)

Figure 5.15: Overall Success Rate (OSR) of Ride Sharing Service (RSS) for Non-Peak Hours

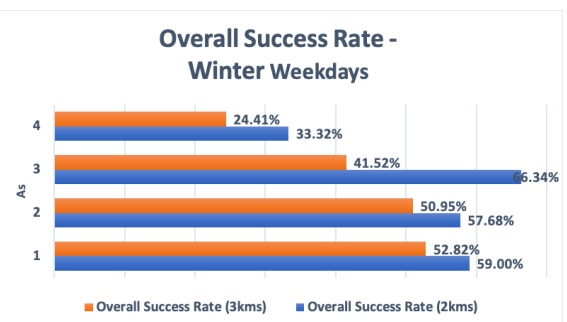

(a)

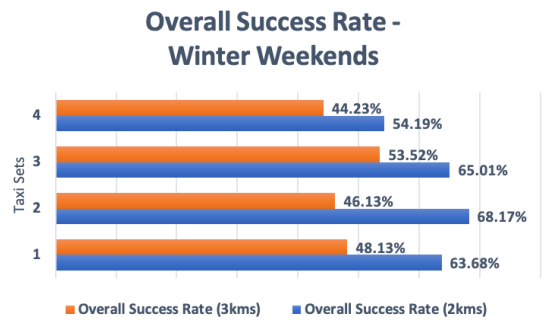

(b)

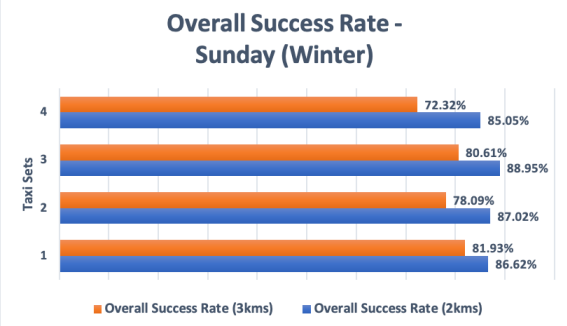

(c)

Figure 5.16: Overall Success Rate (OSR) of Ride Sharing Service (RSS) for Peak Hours

the OSR suffers by increasing the taxi search radius from $2 \mathrm{~km}$ to $3 \mathrm{~km}$, as observed in the figure throughout. A larger taxi radius sends the ride-sharing request to more number of taxis and requires additional computations for ride matching, thus deteriorating the performance of the system.

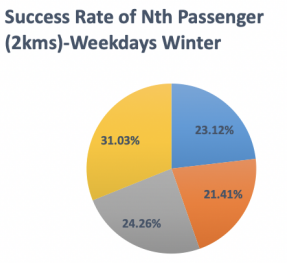

(a) Non-Peak Hours
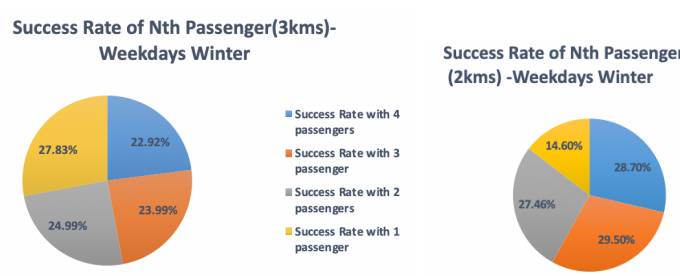

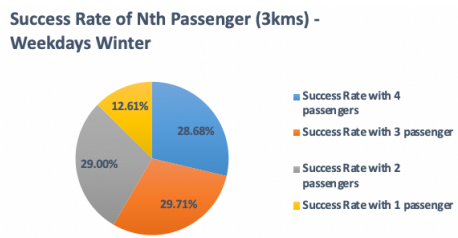

(b) Peak Hours

Figure 5.17: Success Rate of $N^{\text {th }}$ passengers (SNRP) on Weekdays

Fig 5.17, 5.18 and 5.19 show the success rate of $N^{\text {th }}$ passenger (SRNP). Through these figures we can understand how the confirmed rides are allotted to the taxis. We can see if our RSS is matching only single passengers to a taxi or is matching the new passenger requests to taxis with existing passengers en route to their destinations. The figures of the left hand side $(5.17 \mathrm{a}, 5.18 \mathrm{a}$ and 5.19a) are for non-peak hours and we can see that the highest percentage is with 1 passenger. That means more ride requests are matched to taxi for non-shared rides. This pattern is similar for both $2 \mathrm{~km}$ and $3 \mathrm{~km}$ radius. This arises due to sporadic requests during non-peak hours, the RSS tries to match at least one passenger. We see that there is not a very large gap, the remaining rides are almost equally (above 20\%) shared by taxis having 2, 3 and 4 passengers as well. In contrast 
Success Rate of Nth Passenger (2kms) -Weekends Winter

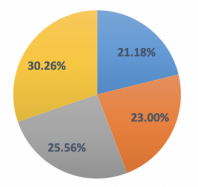

Success Rate of Nth Passenger (3kms) Weekends Winter

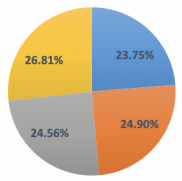

(a) Non-Peak Hours

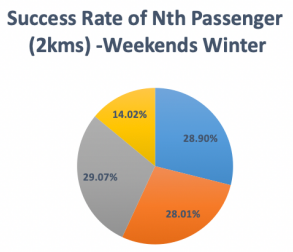

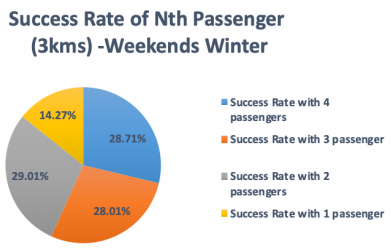

(b) Peak Hours

Figure 5.18: Success Rate of $N^{\text {th }}$ passengers (SNRP) on Weekends

Success Rate of Nth Passenger (2kms) - Sunday(Winter)

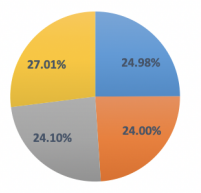

Success Rate of Nth Passenger(3kms) -Sunday (Winter)

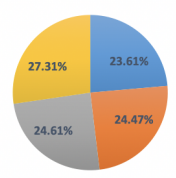

(a) Non-Peak Hours

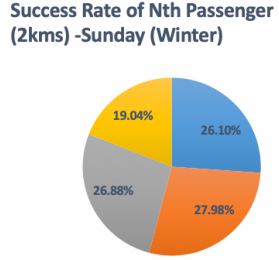

Success Rate of Nth Passenger (3kms) -Sunday (Winter)

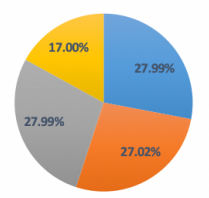

I- Success Rate with 4
passengers (3kms) - Success pate with 3 passenger - Success Rate with 2
passengers In Success Rate with 1 passenger

Figure 5.19: Success Rate of $N^{\text {th }}$ passengers on Sunday

the behaviour of our RSS varies for the peak hours, we can see from the pie chart on the right hand side (5.17b,5.18b and 5.19b), less rides are allotted to taxis with only one passenger. The ride match for 1 passenger only in a taxi is as low as $12.61 \%$. We can also see for the other case i.e., with 2, 3,4 passengers, the OSR is almost similar. This shows that during the peak hours the taxis already occupied by passengers are likely to accept incoming requests. This is because of the fact that, during peak hours more taxis as well are passengers are likely to be present at common locations and are easily matched.

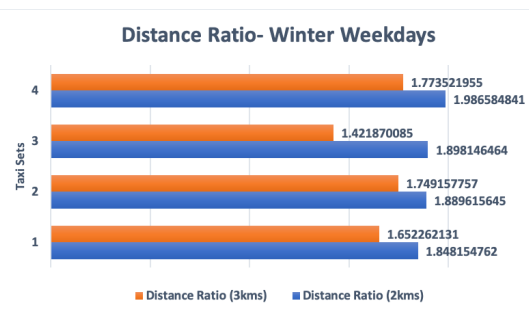

(a)

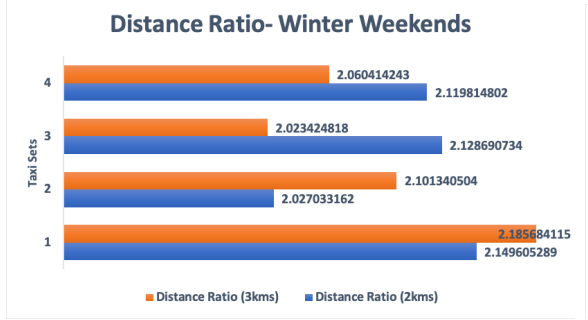

(b)

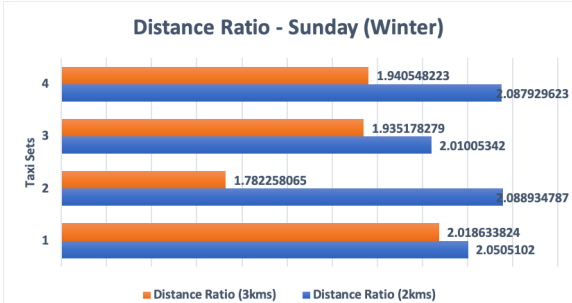

(c)

Figure 5.20: Distance Ratio (DR) for Non-Peak Hours

The results for distance Ratio (DR) is captured in Fig 5.20 for the non-peak hours. We notice a consistent behaviour for all the three categories as well as the different combination of taxi sets with $2 \mathrm{~km}$ and $3 \mathrm{~km}$ radius. The DR value correlates to the extra travelling distance for shared rides compared to single rides. Thus a higher value for the distance ratio shows that there is a significant improvement on the total distance travelled with multiple passengers sharing their ride 


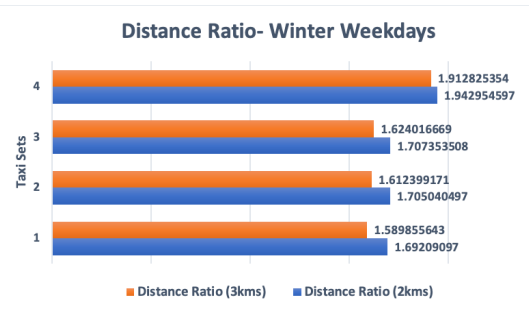

(a)

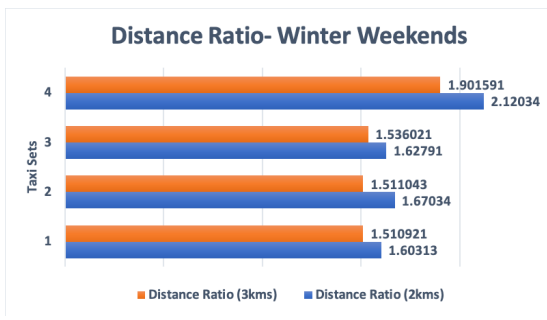

(b)

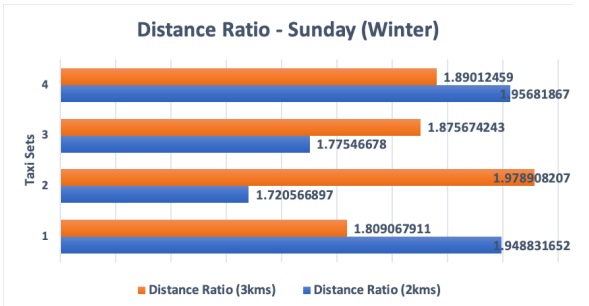

(c)

Figure 5.21: Distance Ratio (DR) for Peak Hours

versus single rides. For both weekdays 5.20a and weekends 5.20b, the DR value is consistently better for taxi search radius within $2 \mathrm{~km}$. We can infer that if the taxis are nearer to the ride requests, there will be smaller deviation from their original path. Thus we obtain a better result for $2 \mathrm{~km}$ taxi search radius. For Sundays, as seen in 5.20c, the behaviour is consistent for taxi sets 1 , 2 and 3, however for taxi set 4, the DR for $3 \mathrm{~km}$ performs better. The reason can be due to better positioning of taxis and similar routes for the data set on the particular date. The results for the peak hours are consistent with our observations for non-peak hours as seen in Fig 5.21a and Fig 5.21b. However, the pattern for Sunday is not consistent with our previous inferences as seen in Fig 5.21c. This is inferred to be caused by a different travelling habits of people during Sundays as compared to other weekdays or weekends. On Sundays people generally tend to stay in their homes in the earlier half, and may go out to church or public places with their families.

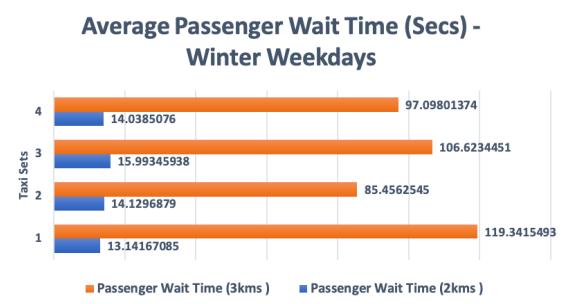

(a)

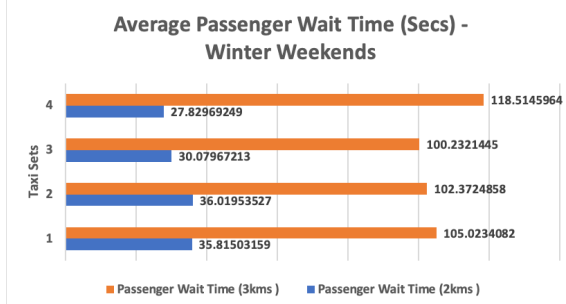

(b)

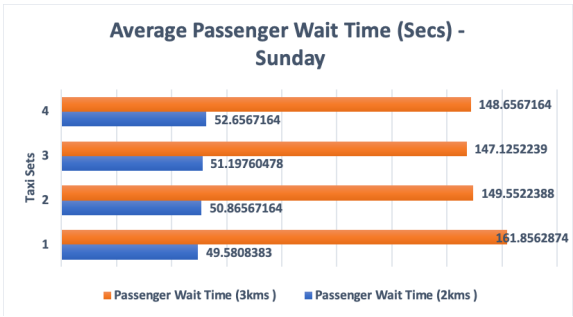

(c)

Figure 5.22: Average Passenger Wait Time (AWT) in Seconds for Non-Peak Hours

The average passenger wait time (AWT) for both non-peak and peak hours are captured in Fig 5.22 and Fig 5.23,respectively. We observe a small improvement in the AWT in case of taxi set 4 compared to the other taxi sets. This means that AWT improves when served by taxi sets that is twice the original set of taxi. However, this is not the case with peak hours, the AWT value drops (smaller value preferred) for taxi sets 2,3 which means the average waiting time is improved when taxis are injected to spots of low and high demands. This shows that additional taxis improves the chances of serving the incoming requests faster. However, we also notice a sharp increase in AWT when the taxi search radius is increased to $3 \mathrm{~km}$. This means that the taxis at a further distance can serve the ride requests but will require additional time to reach the passenger to pick them up as compared to the taxis within $2 \mathrm{~km}$ radius. 


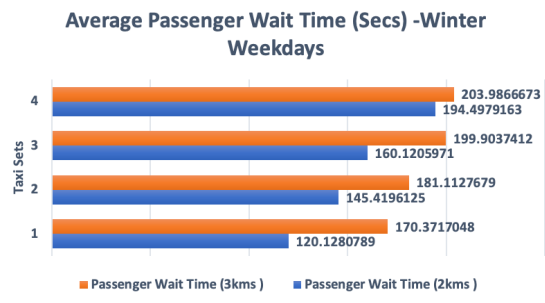

(a)

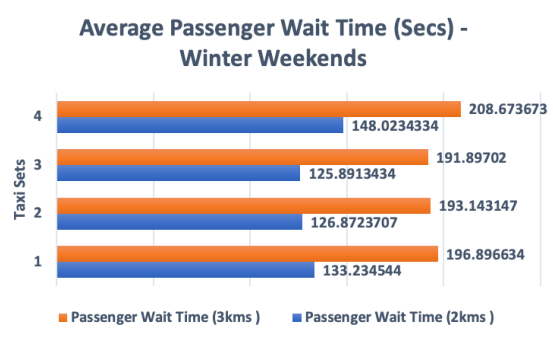

(b)

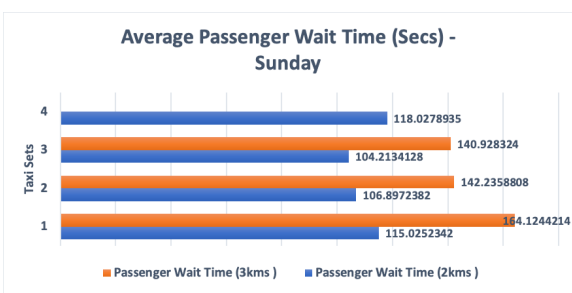

(c)

Figure 5.23: Average Passenger Wait Time (AWT) in Seconds for Peak Hours

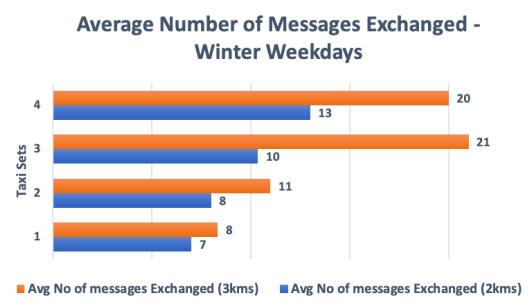

(a)

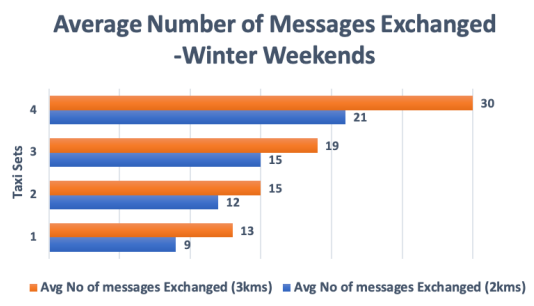

(b)

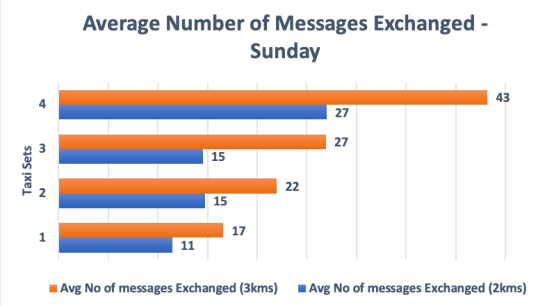

(c)

Figure 5.24: Average Number of Messages Exchanged (ANM) for Non-Peak Hours

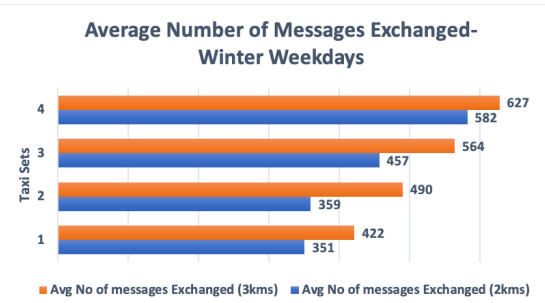

(a)

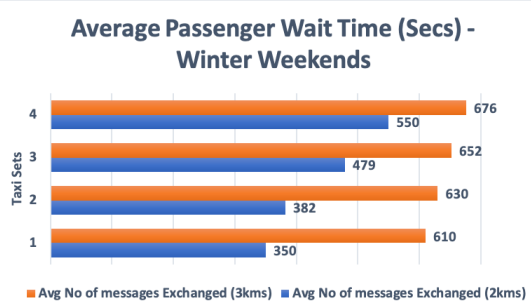

(b)

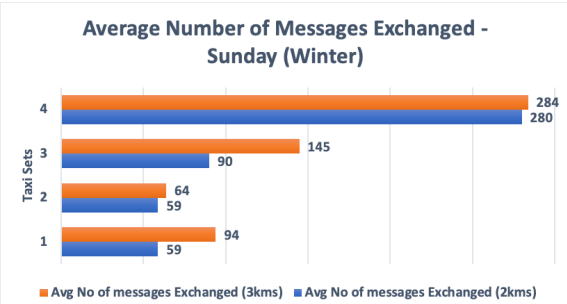

(c)

Figure 5.25: Average Number of Messages Exchanged (ANM) for Peak Hours

The average number of messages (ANM) exchanged in the RSS is as low as 6 to a maximum of 43 for non-peak hours as shown in Figures 5.24a 5.24b and 5.24c. With an increase in the number of taxis from taxi set 1 to 4, we notice the ANM also increases. Further the ANM for $3 \mathrm{~km}$ is more than for $2 \mathrm{~km}$ radius throughout the results obtained. This is because more taxis are available at a larger radius and hence more messages are sent back and forth to confirm the rides. From Fig 5.25, it is seen that ANM has much larger values as compared to non-peak hours, but even in this case the ANM value increases with more number of taxis as seen in taxi set 2, 3 and 4 . The minimum value for peak hour is observed at 351 for taxi set 1 during the weekdays and the maximum value is 627 for taxi set 4 with a $3 \mathrm{~km}$ taxi radius. The huge number of ANM is due to the large number of ride requests and equally larger number of taxis available to receive the ride requests.

The average request processing time (ARPT) is for non-peak hours is shown in Fig 5.26 and for peak hours in Fig 5.27. It can be noticed from the figures that there are huge differences in 


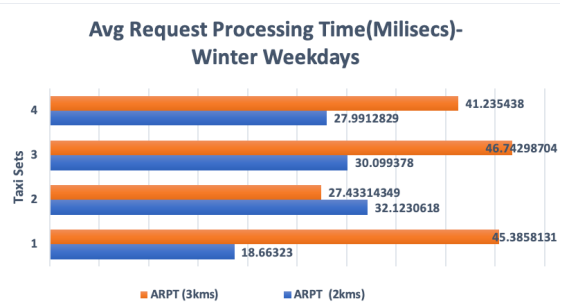

(a)

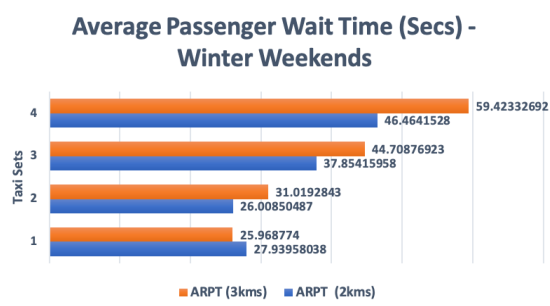

(b)

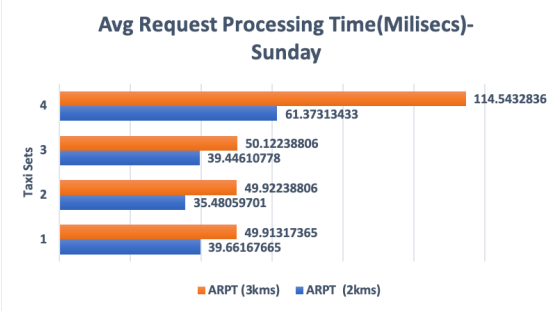

(c)

Figure 5.26: Average Request Processing Time (ARPT) in Milliseconds for Non-Peak Hours

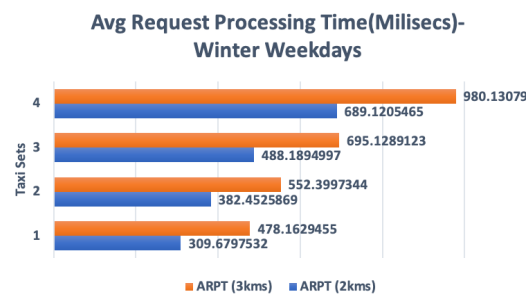

(a)

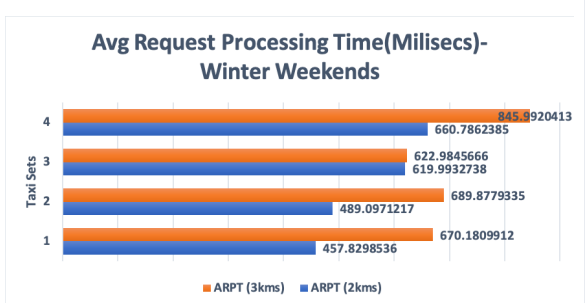

(b)

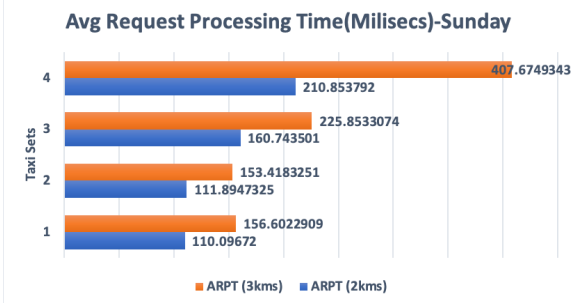

(c)

Figure 5.27: Average Request Processing Time (ARPT) in Milliseconds for Peak Hours

ARPT values between different hours. Smaller ARPT values are observed for non peak hours with a minimum of $18.67 \mathrm{~ms}$. The increase in value occurs both due to additional taxis as well as increase in the taxi search radius. Similarly, an almost identical trend is observed for peak hours in terms of the growth in values for various taxi sets. However, the huge values of ARPT which ranges between $310-980 \mathrm{~ms}$ is understood to be due to the large number of requests which utilizes more resources for the complex computations and hence takes more time to process the requests. Thus, resulting into much higher values of ARPT. However, we can observe here that the actual request processing time is minimal (in milliseconds), which shows that our application provides very quick response for the experimental setup chosen in this case. 


\subsection{Summary of the observations}

In conclusion, the behavior of the RSS is different based on different days of the week as well as for different hours (peak and non-peak). But for both the peak and non-peak hours in the week, the overall success rate is improved by injecting more taxis at the locations having more ride requests. However, in case of peak hours, inserting too many taxis (taxi set 4 which is double the initial number of taxis) hampers the performance of the RSS. The increase in the number of taxis either by injecting the taxis in the different sets of taxis (1 through 4) as well as by increasing the taxi search radius impacts the system, which decreases the distance ratio (higher value desired), increases the average passenger wait time, increases the number of messages exchanged in the system, and also increases the average request processing time. Therefore, the performance of the system can be improved by carefully examining the direct correlation of the performance matrices as defined in Section 5.2 and changing the values accordingly. From the graphs plotted for both summer and winter weeks, we can gather that the pattern does not change drastically. The patterns observed for weekdays, weekends and Sundays for both the seasons are overall similar with a slight variation in the values. 
Table 5.2: Comparison of RSS using PSO and TRS Algorithm

\begin{tabular}{|c|c|c|c|c|}
\hline \multirow{2}{*}{ Parameters } & \multicolumn{2}{c|}{ RSS using PSO } & \multicolumn{2}{c|}{ TRS Algorithm } \\
& Non-Peak Hour & Peak Hour & Non-Peak Hour & Peak Hour \\
\hline No of Requests & 121 & 1896 & 121 & 1896 \\
\hline No of Taxis (Set 1) & 37 & 490 & 37 & 490 \\
\hline Overall Success Rate of Ride Matching (OSR) \% & 91.74 & 63.29 & 32.4 & 19.7 \\
\hline Success Rate Nth Passengers (N=4) \% & 23.42 & 28 & 21.01 & 12.17 \\
\hline Success Rate Nth Passengers (N=3) \% & 23.42 & 28.42 & 20.34 & 28.04 \\
\hline Success Rate Nth Passengers (N=2) \% & 25.23 & 28.42 & 25.53 & 29.25 \\
\hline Success Rate Nth Passengers (N=1) \% & 27.93 & 15.17 & 33.12 & 30.54 \\
\hline Distance Ratio (DR) & 1.87 & 1.73 & 0.96 & 0.6 \\
\hline Average Waiting Time (AWT) in secs & 17.14 & 120.13 & 160.72 & 500.72 \\
\hline Average Number of Messages (ANM) & 5 & 269 & 21 & 1076 \\
\hline Average Request Processing Time (ARPT) in millisecs & 13.81 & 361.91 & 2000.19 & 16000.67 \\
\hline
\end{tabular}

\subsection{Comparison of Results}

In this section, we compare the results obtained using our method to the results obtained using the Taxi Ride Sharing (TRS) algorithm [11]. We have implemented the approach described in Batla et al. [11]. They proposed a distributed algorithm for ridesharing that used localized communication among passengers and nearby taxi drivers. They have tested the algorithm with a large scale GPS traces of 4,000 taxis in Shanghai, China, as we have done with a large Chicago data set. Their work has similar goals to the our method such as an increase in shared rides for the taxis, as well as cost-effectiveness for both taxis and passengers. Our RSS is an extension of the algorithms and metrics designed in their work.

The scheme planned to handle incoming requests sequentially, thus the request processing time was longer, and the overall performance worsened. In contradiction, our method handles requests in a parallel manner, which helps to reduce the waiting time and improve the performance of the ride-sharing system. For comparison, we implemented the TRS algorithm for non-peak and peak hours and compared the results with our algorithm as shown in Table 5.2. The comparison is based on the various performance metrics that we have defined in previous sections.

We compare our PSO implementation with TRS for both peak and non-peak hours with a similar number of requests and taxis available. From the table, we can see that the overall success rate of ride-matching (OSR) is $91.74 \%$ for non-peak hours compared to TRS which is $32.4 \%$. For peak hours we see a lower OSR of $63.29 \%$, but it is much higher than the TSR having 19.7\%. This indicates that RSS using PSO does a better job of matching the ride requests with the available taxis. The SRNP determines how well the ride requests are handled by matching them as ride-shares and by channeling incoming ride requests to taxis that are already matched with passengers sharing similar routes. We have collected data for taxis that are matched with 4, 3, 2 and 1 passenger. Our goal is to maximize the ride-share by having higher numbers for SNRP with more than 1 passenger. From the table, we can see a higher SNRP is achieved for both peak and non-peak hours using RSS. For N=4, during non-peak hours, we see an SNRP of $23.42 \%$ compared to $21.01 \%$ for TRS. In this case, our method does slightly better than the TRS method. While there is a drastic difference during peak hours, with an SNRP of $28 \%$ compared to $12.17 \%$ of TRS. As discussed 
previously, RSS with PSO is designed to better the chances of a match with taxis already having passengers to facilitate ride-sharing, thus this figure reflects the goal that we are trying to achieve. We observed that there is no such design in TRS and the incoming requests are matched solely based on the capability of the algorithm to match the nearest available taxi.

Similar observations were recorded for $\mathrm{N}=3$, however, we see a different trend for SNRP with $\mathrm{N}=2,1$. For $\mathrm{N}=2$, we see that a small difference of values such as $25.23 \%$ for non-peak hours for RSS with PSO versus $25.53 \%$ for TRS, and $28.42 \%$ for peak-hours versus $29.25 \%$. These figures indicate that our algorithm tries to match rides with taxis having more than 2 passengers to improve the ride-sharing and help passengers benefit from it. We see a drastic change in the SNRP for $\mathrm{N}=1$, which are taxis matched to single passengers and hence is not a shared ride. The figures obtained for non-peak time is $27.93 \%$ using RSS with PSO versus $33.12 \%$ and $15.17 \%$ versus $30.54 \%$ for peak hours. There are two major takeaways from these observations. Firstly, we again notice that the RSS with PSO tries not to match rides to single taxis and it does a better job during the peak hours. Secondly, the SNRP obtained for TRS with rides matched to a single ride is higher than with more passengers. We see $33.12 \%$ and $30.54 \%$ as the highest readings among their respective categories. This indicates that although TRS does a better job at matching individual passenger to a taxi, which further lowers its chances of ride-sharing.

Subsequent is the distance ratio (DR) which shows the economy of the distance traveled by taxi with single rides in contrast to shared rides. A higher ratio is desired and we can see for both nonpeak and peak hours, 1.87 and 1.73 is obtained using RSS with PSO. Comparatively, lower values are obtained in the case of TRS (less than 1). We can also observe a lower average of messages (5- non-peak and 269- peak) being passed in our system as opposed to TRS (21-non-peak and 1076-peak). A lower number of messages can be beneficial as it does not overload the system and slow down the performance. Rides are efficiently mapped with a lesser number of messages in RSS using PSO. Finally, the ARPT is also drastically different, such as $13.81 \mathrm{~ms}$ for the non-peak hour and $361.91 \mathrm{~ms}$ with RSS using PSO. While a comparably higher value is obtained using TRS, $2000.19 \mathrm{~ms}$, and $16000.67 \mathrm{~ms}$ for non-peak and peak hours. This metric indicates that RSS using PSO is much faster in processing requests to provide better ride-share matching.

In conclusion, TRS does a good job of reducing the total distance traveled.It however, does not tackle the problem of distributing rides more evenly and tends to match single requests and taxis. This approach did not necessarily aid effective ride-sharing. 


\section{Chapter 6 : Conclusion and Future Works}

In this thesis, we proposed a dynamic and hybrid model using an evolutionary (heuristic) algorithm for the ride-sharing system (RSS). The objective is to have a better ride-matching capability among shared vehicles, reduce response time from the taxis as well as the overall distance traveled by the taxis to fulfill the shared rides. In the next section, we will summarize the results obtained.

\subsection{Summary}

The RSS system has been tested using real-world data set of taxis for the city of Chicago. We have performed experiments using a separate data sets for summer and winter weeks. We evaluated the performance of the algorithms using the various metrics and the constarints that we defined.

Based on the experimental results, it can be concluded that our RSS can provide ride-sharing services to several passengers and simultaneously minimize the distance traveled by the taxis, thereby reducing the commute time and the carbon footprint in the environment. We compared the results obtained using the RSS algorithm and using the TRS algorithm [11]. The overall success rate (OSR) for non-peak hours obtained by using RSS was $91.74 \%$ which is approximately 3 times better than $32.4 \%$, which is the value obtained using TRS. Similarly, for peak-hours RSS was able to achieve $63.29 \%$ as compared to $19.7 \%$ for TRS.

\subsection{Future Works}

In the future, an extension of our algorithm with an added theoretical analysis of the influences of asynchrony on ride-sharing can be done. Also, the same approach can be used with different heuristic algorithms to study the outcomes. More recent data and larger datasets of different cities can be used to evaluate the Success Rate (SRNP) of the $N^{\text {th }}$ passenger in greater detail. Research can be done to address various open challenges is the ride-sharing area such as incorporating autonomous vehicles in a ride-sharing system, useful customer feedback system, developing faulttolerant systems. We also plan to model road congestion for better ride-share route proposition to improve the profitability of the taxi drivers and reduced journey time for the passengers. An efficient revenue model for ride-sharing business solutions can also be explored further. 


\section{References}

[1] Shrawani Silwal, Md Osman Gani, and Vaskar Raychoudhury. A survey of taxi ride sharing system architectures. In 2019 IEEE International Conference on Smart Computing (SMARTCOMP), pages 144-149. IEEE, 2019.

[2] Niels Agatz, Alan Erera, Martin Savelsbergh, and Xing Wang. Optimization for dynamic ride-sharing: A review. European Journal of Operational Research, 223(2):295-303, 2012.

[3] Roohollah Kalatehjari, A Rashid, Ahmad Safuan, Nazri Ali, and Mohsen Hajihassani. The contribution of particle swarm optimization to three-dimensional slope stability analysis. The Scientific World Journal, 2014, 2014.

[4] Weicheng Zhao, Yajuan Qin, Dong Yang, Linjuan Zhang, and Wanting Zhu. Social group architecture based distributed ride-sharing service in vanet. International Journal of Distributed Sensor Networks, 10(3):650923, 2014.

[5] Shuo Ma, Yu Zheng, and Ouri Wolfson. Real-time city-scale taxi ridesharing. IEEE Transactions on Knowledge and Data Engineering, 27(7):1782-1795, 2015.

[6] Pedro M d'Orey, Ricardo Fernandes, and Michel Ferreira. Empirical evaluation of a dynamic and distributed taxi-sharing system. In 2012 15th International IEEE Conference on Intelligent Transportation Systems, pages 140-146. IEEE, 2012.

[7] Haoxiang Yu, Vaskar Raychoudhury, and Shrawani Silwal. Dynamic taxi ride sharing using localized communication. In Proceedings of the 21st International Conference on Distributed Computing and Networking, pages 1-10, 2020.

[8] FHWA. National Household Travel Survey, 2017. Accessed on 06.06.2018.

[9] City of New York. Nyc taxi limousine commission. https://www1.nyc.gov/site/tlc/ about/tlc-trip-record-data.page, 2019.

[10] City of San Francisco. Datasf. https://datasf .org/opendata/, 2019.

[11] Kanika Bathla, Vaskar Raychoudhury, Divya Saxena, and Ajay D Kshemkalyani. Real-time distributed taxi ride sharing. In 201821 st International Conference on Intelligent Transportation Systems (ITSC), pages 2044-2051. IEEE, 2018.

[12] Chicago Data Portal. Chicago data portal. https://data.cityof chicago.org/, 2017.

[13] Pedro M d'Orey, Ricardo Fernandes, and Michel Ferreira. Reducing the environmental impact of taxi operation: the taxi-sharing use case. In 2012 12th International Conference on ITS Telecommunications, pages 319-323. IEEE, 2012. 
[14] Jeffery B Greenblatt and Susan Shaheen. Automated vehicles, on-demand mobility, and environmental impacts. Current sustainable/renewable energy reports, 2(3):74-81, 2015.

[15] Harilaos N Psaraftis. A dynamic programming solution to the single vehicle many-to-many immediate request dial-a-ride problem. Transportation Science, 14(2):130-154, 1980.

[16] Deb Kalyanmoy. Multi objective optimization using evolutionary algorithms. John Wiley and Sons, 2001.

[17] Russell Eberhart and James Kennedy. Particle swarm optimization. In Proceedings of the IEEE international conference on neural networks, volume 4, pages 1942-1948. Citeseer, 1995.

[18] Roberto Baldacci, Vittorio Maniezzo, and Aristide Mingozzi. An exact method for the car pooling problem based on lagrangean column generation. Operations Research, 52(3):422439, 2004.

[19] Jiaqi Ma, Xiaopeng Li, Fang Zhou, and Wei Hao. Designing optimal autonomous vehicle sharing and reservation systems: A linear programming approach. Transportation Research Part C: Emerging Technologies, 84:124-141, 2017.

[20] Jean-François Cordeau. A branch-and-cut algorithm for the dial-a-ride problem. Operations Research, 54(3):573-586, 2006.

[21] Ahmed Atahran, Christophe Lenté, and Vincent T'kindt. A multicriteria dial-a-ride problem with an ecological measure and heterogeneous vehicles. Journal of Multi-Criteria Decision Analysis, 21(5-6):279-298, 2014.

[22] Zhihai Xiang, Chengbin Chu, and Haoxun Chen. A fast heuristic for solving a large-scale static dial-a-ride problem under complex constraints. European journal of operational research, 174(2):1117-1139, 2006.

[23] Yeqian Lin, Wenquan Li, Feng Qiu, and He Xu. Research on optimization of vehicle routing problem for ride-sharing taxi. Procedia-Social and Behavioral Sciences, 43:494-502, 2012.

[24] Shuo Ma, Yu Zheng, and Ouri Wolfson. T-share: A large-scale dynamic taxi ridesharing service. In 2013 IEEE 29th International Conference on Data Engineering (ICDE), pages 410-421. IEEE, 2013.

[25] Jianren Gao, Yuxin Wang, Haoyang Tang, Zhao Yin, Lei Ni, and Yanming Shen. An efficient dynamic ridesharing algorithm. In 2017 IEEE International Conference on Computer and Information Technology (CIT), pages 320-325. IEEE, 2017.

[26] Claudia Archetti, Martin Savelsbergh, and M Grazia Speranza. The vehicle routing problem with occasional drivers. European Journal of Operational Research, 254(2):472-480, 2016. 
[27] Mengyang Liu, Zhixing Luo, and Andrew Lim. A branch-and-cut algorithm for a realistic dial-a-ride problem. Transportation Research Part B: Methodological, 81:267-288, 2015.

[28] Niels Agatz, Alan L Erera, Martin WP Savelsbergh, and Xing Wang. Dynamic ride-sharing: A simulation study in metro atlanta. Procedia-Social and Behavioral Sciences, 17:532-550, 2011.

[29] Xing Wang, Niels Agatz, and Alan Erera. Stable matching for dynamic ride-sharing systems. Transportation Science, 52(4):850-867, 2017.

[30] Ming Zhu, Xiao-Yang Liu, and Xiaodong Wang. An online ride-sharing path-planning strategy for public vehicle systems. IEEE Transactions on Intelligent Transportation Systems, (99):1-12, 2018.

[31] Yongzheng Jia, Wei Xu, and Xue Liu. An optimization framework for online ride-sharing markets. In 2017 IEEE 37th International Conference on Distributed Computing Systems (ICDCS), pages 826-835. IEEE, 2017.

[32] Wei Lu. Optimization and mechanism design for ridesharing services. PhD thesis, 2015.

[33] Bin Cao, Louai Alarabi, Mohamed F Mokbel, and Anas Basalamah. Sharek: A scalable dynamic ride sharing system. In 2015 16th IEEE International Conference on Mobile Data Management, volume 1, pages 4-13. IEEE, 2015.

[34] Junqi Ning, Rong Chen, and Zhifeng Zhou. Improving large scale real-time ridesharing with heuristics for road networks. In 2018 IEEE SmartWorld, Ubiquitous Intelligence \& Computing, Advanced \& Trusted Computing, Scalable Computing \& Communications, Cloud \& Big Data Computing, Internet of People and Smart City Innovation (SmartWorld/SCALCOM/UIC/ATC/CBDCom/IOP/SCI), pages 515-521. IEEE, 2018.

[35] Yazhe Wang, Baihua Zheng, and Ee-Peng Lim. Understanding the effects of taxi ridesharinga case study of singapore. Computers, Environment and Urban Systems, 69:124-132, 2018.

[36] Javier Alonso-Mora, Samitha Samaranayake, Alex Wallar, Emilio Frazzoli, and Daniela Rus. On-demand high-capacity ride-sharing via dynamic trip-vehicle assignment. Proceedings of the National Academy of Sciences, 114(3):462-467, 2017.

[37] Gerardo Berbeglia, Jean-François Cordeau, and Gilbert Laporte. A hybrid tabu search and constraint programming algorithm for the dynamic dial-a-ride problem. INFORMS Journal on Computing, 24(3):343-355, 2012.

[38] Rui Chen and Christos G Cassandras. Optimization of ride sharing systems using eventdriven receding horizon control. arXiv preprint arXiv:1901.01919, 2019. 
[39] Mitja Stiglic, Niels Agatz, Martin Savelsbergh, and Mirko Gradisar. Enhancing urban mobility: Integrating ride-sharing and public transit. Computers \& Operations Research, 90:12-21, 2018.

[40] Pradip Kumar Sharma, Seo Yeon Moon, and Jong Hyuk Park. Block-vn: A distributed blockchain based vehicular network architecture in smart city. JIPS, 13(1):184-195, 2017.

[41] Dejan Dimitrijević, Vladimir Dimitrieski, and Nemanja Nedić. Prototype implementation of a scalable real-time dynamic carpooling and ride-sharing application. Informatica, 38(3), 2014.

[42] Masayo Ota, Huy Vo, Claudio Silva, and Juliana Freire. A scalable approach for data-driven taxi ride-sharing simulation. In 2015 IEEE International Conference on Big Data (Big Data), pages 888-897. IEEE, 2015.

[43] Mustafa Lokhandwala and Hua Cai. Dynamic ride sharing using traditional taxis and shared autonomous taxis: A case study of nyc. Transportation Research Part C: Emerging Technologies, 97:45-60, 2018.

[44] Fu-Shiung Hsieh, Fu-Min Zhan, and Yi-Hong Guo. A solution methodology for carpooling systems based on double auctions and cooperative coevolutionary particle swarms. Applied Intelligence, 49(2):741-763, 2019.

[45] United States Census Bureau. QuickFacts Chicago City, Illinois. Accessed on 03.03.2019.

[46] Google. Google. https://cloud.google.com/maps-platform/, 2017.

[47] HERE. Here. https://developer .here.com, 2019.

[48] Inc Postman. Postman. https ://www.getpostman.com/, 2019. 